\title{
Effect of geometrical contraction on vortex breakdown of swirling turbulent flow in a model combustor
}

\author{
Y. Wu, C. Carlsson, R. Szasz, L. Peng, L. Fuchs, X. S. Bai* \\ Div. Fluid Mechanics, Lund University, Lund, Sweden
}

\begin{abstract}
Large Eddy Simulation (LES) studies of isothermal and incompressible turbulent swirling flows in a model gas turbine combustion chamber geometry have been carried out. The focus is on the effect of outlet geometry contraction on the vortex breakdown structure and the precessing vortex core in the chamber. Nine different outlet geometries with different contraction ratio $\mathrm{C}_{r}$ are considered. The results from a baseline case are compared with experimental data in the literature. The swirling flow is generated using a swirler with fifteen guide vanes similar to an existing industrial gas turbine burner. In all cases the swirler and the main chamber geometry are kept the same. The detailed swirler geometry is considered in the simulation using unstructured grids. Sensitivity tests on the influence of the grid resolution and the subgrid scale models are carried out. The mean flow field shows different vortex breakdown structures when the contraction ratio changes from 0.325 to 1.0 . In particular, along the axis of the chamber the flow is shown to switch its direction when the contraction increases as a result of the change of the structure of the center recirculation zone. The underlying flow physics is analysed by comparing the budget terms in the momentum equations, and by performing a global instability analysis.
\end{abstract}

Keywords: Swirling flow, vortex breakdown, gas turbine combustor, outlet contraction

\footnotetext{
*Corresponding author, Xue-Song.Bai@energy.lth.se
} 


\section{Introduction}

In combustion devices, the geometry at the combustor inlet is typically expanding whereas at the outlet it is contracting. When a swirling flow is employed to supply the fuel and the air, such a combustion chamber geometry results in a distinct vortex breakdown structure, in which vortex breakdown is not only a function of the swirl number and the swirl velocity profile but also the inlet/combustor expansion ratio, outlet/combustor contraction ratio, and combustor length/diameter ratio. A sudden expansion of the inlet section leads to a sudden expansion of the flow, which results in the formation of corner vortices that interact with the center vortex breakdown region forming a complex flow structure [1]. Contraction at the combustion chamber exit with a convergent angle or with a sudden contraction leads to acceleration of the flow producing a favourable pressure gradient that can eliminate the adverse pressure gradient promoted by the upstream swirling flow. In certain cases, the contraction outflow is strong enough to cause significant change in the size and location of the vortex breakdown bubble.

The effect of outlet contractions on swirling flows has been studied experimentally by different groups. Escudier, Bornstein, and Zehnder [2] reported experiments on the effect of outlet contraction on the vortex core, in which five cases have been considered with the contraction ratios $d / D=$ $10 / 55,18 / 55,25 / 55,40 / 55,55 / 55$, where $D$ and $d$ are respectively the main chamber diameter and the exhaust pipe diameter. The Reynolds numbers in these cases are low; the low contraction ratio cases $(d / D=40 / 55$ and $55 / 55)$ have a Reynolds number of 4800, and the other three cases have a Reynolds number of 1900. It was found that the axial velocity was reversed at the core part for the $d / D=40 / 55$ and $55 / 55$ cases, while this reversed flow disappeared in the remaining smaller $d / D$ cases; the core is also much thicker and relatively straight for the large $d / D$ cases compared with the smaller ones. Similar effects of the outlet contraction on the vortex breakdown structures have been observed in a recent experimental study of swirling isothermal and reactive flows in a combustor geometry for higher Reynolds number turbulent flows [3]. In some other studies the outlet contraction has shown low impact on the flow structures in the main chamber under a condition that the swirl number is relatively low and the chamber length to diameter ratio is relatively large $[4,5]$.

To explain the onset of vortex breakdown, Benjamin [6] based on the the-

ory of Squire [7] proposed a flow-state theory based on a subcritical state and 
a conjugate supercritical state. In the subcritical state a wave can propagate to upstream, in the sense that infinitesimal axisymmetric standing waves can occur. In the conjugate supercritical state the standing wave can propagate to downstream. The subcritical state always has a greater momentum flux or flow force than the supercritical state. The breakdown is believed to be not caused by the existence of waves, but rather is the leading wave for weak transitions. The effect of outlet contraction on vortex breakdown has been explained using the flow-state theory. Escudier and Keller [8] examined the effect of contraction ratio at two different guiding vane angle cases (hence the swirl number); the first case has an angle of 62 degrees and the second case with an angle of 70 degrees. The Reynolds number was kept at about 7000 . Under the first vane angle condition (the relatively lower swirl number case) the flow remained supercritical after vortex breakdown when the contraction was used; at the 70 degree vane angle condition (the higher swirl number case), the flow was subcritical with all three contraction ratios used. As a consequence, under the higher vane angle condition, the high contraction ratio case exhibited a jet-like axial velocity and higher maximum tangential velocity, and the recirculation zone assumed a mushroom shape. In a later work, LDV measurements were carried out by Escudier, Nickson, and Poole [9] to measure the mean axial and swirl components of velocity in a swirling turbulent pipe flow at different swirl numbers and contraction conditions. At a low swirl condition (swirl number $\approx 0.3$ ), the flow is supercritical; a closed recirculation bubble vortex breakdown was formed on the axis just downstream of the inlet. At a high swirl condition (swirl number $\approx 0.6$ ), the flow with and without contraction are completely different. With the contraction in place, a closed recirculation region is found centered on the axis but considerably distorted when compared with the elongated smooth shape of the low-swirl vortex breakdown.

To study the effect of outlet contraction on the vortex breakdown and other aspects of the flow physics, e.g. the dynamics of the flow, it is desirable to perform high fidelity numerical simulations, since the simulations can provide more details about the spatial (three-dimensional) and temporal evolution of the flow. Three types of numerical simulations may be employed to study turbulent flows. These are direct numerical simulation (DNS), large eddy simulation (LES), and simulations based on the Reynolds averaged Navier-Stokes (RANS) equations. In DNS the full set of governing equations are numerically solved with a fine spatial and temporal resolution to capture all length and time scale in the flow. Currently, DNS is limited 
to low Reynolds number flows and simple flow geometry. Among the three approaches RANS simulations are the least computational demanding since only the main flow scales are considered in the simulations, which require low spatial and temporal resolution. Speziale et al. [10] showed that RANS simulations are sometimes not capable in predicting the main physical features of turbulent swirling flows, e.g., the flow in an axially rotating pipe.

LES is an approach that is less demanding computationally than DNS but requires more computational effort than RANS. Compared with RANS, LES has an advantage [11] in numerical simulations of turbulent swirling flows containing relatively large and slow fluctuating structures. With LES one resolves the energy-containing large eddies and the large scale (nonturbulent) coherent structures such as the vortex breakdown process and the motion of precessing vortex core (PVC). For a very complex gas turbine combustor geometry, Selle et al. [12] demonstrated the capacity of LES to predict the mean flow, with and without combustion, as well as its main unstable modes. Most of the unsteady motion residing in the large structures was very well predicted by the LES approach.

Wegner et al. [13] compared the results from unsteady RANS (URANS) and LES for predicting swirl flow instability; it was found that a good agreement between the mean velocities can be achieved when comparing the URANS results with the experimental data and LES results; however, it was also found that the energy contained in the coherent motion of the precessing vortex core was significantly under-predicted in the unsteady RANS. Computational requirements for an unsteady RANS are still low when compared with those for an LES study. LES is very good at capturing both the mean flow field and the large unsteady coherent structures, which can be captured even with a fairly coarse mesh.

Nickolaus and Smith [14] also performed RANS and LES in analysis of highly swirled and turbulent flows in a dump combustor. LES was shown to capture more accurately the flow features than RANS did. However, LES studies need more computational time to achieve convergence of the mean field than RANS. The simulations were carried out on the same mesh, with the LES running for 3 weeks, while the RANS model required only one day for convergence.

Escue and Cui [15] presented a comparison between experimental results and RANS simulations of swirling turbulent flows; they reported that for lower Rossby numbers ( $R o \leqslant 1$ ), the re-normalization group (RNG) $k-\varepsilon$ model was superior to the Reynolds stress transport model. However, as the 
Ro was increased to 2, none of these models were shown to perform well.

This paper reports on a LES study of the effect of outlet contraction on the structures and dynamics of swirling turbulent flows in a model combustion chamber geometry equipped with an industrial gas turbine burner. Laser doppler velocimetry measurements of the flow field have been reported earlier by Orbay et al. [3]. LES of this flow case with and without the outlet contraction was also reported previously [3]. Numerical results indicated that owing to the conservation of the angular momentum the swirl/tangential velocity is increased by the contraction, which affects the radial pressure gradient and subsequently the entire flow structure. However, it is unclear how the vortex breakdown structures develop with respect to the variation in the outlet contraction, e.g. if there is a bifurcation process involved in the transition from center bubble type vortex breakdown to an annular breakdown. To investigate this, nine cases are simulated with different outlet contractions but with the same swirler and combustion chamber geometry. Another feature of this study is that we consider the full geometry of the swirler in the simulation, which is made up of 15 guide vanes. The previous LES study [3] has neglected the full details of the swirler geometry but used the experimental data at the burner exit plane as the inflow boundary condition.

LES generates a huge database that contains not only the information about the mean and the variance of the turbulent flow but also information about the dynamics and the coherent structures of the flow. The frequency specific flow motion at a given flow field point can be analyzed by means of the Fourier transform; however, it is difficult to relate point specific frequencies to the spatial structures. Proper orthogonal decomposition (POD) $[16,17]$ is a method by which the original (high dimensional) flow data can be projected into a lower-dimensional space and as such relevant flow structures hidden in the original data can be extracted. Frequencies of extracted high energy containing flow structures can be obtained through a Fourier transform of POD time coefficients. More recently, dynamic mode decomposition (DMD) [18, 19], a method connected to POD, has been developed for extracting temporal correlation, where frequency specific spatial structures can be directly distinguished from time and spatially resolved data. DMD has been applied in combustion environments for elucidation of the effect of burner geometry on PVC [20] and flame/flow interaction in swirling flames [21]. POD and DMD are used here to analyze the effect of outlet contraction on the dynamic motion of the flow.

The main goal of this study is to systematically investigate the effect of 
outlet contraction on the vortex break process and on the dynamics of PVC in a model gas turbine combustor studied earlier [3]. The second goal is to examine the sensitivity of LES results to the detailed swirler geometry, the subgrid scale (SGS) models, and the grid resolution. It is found that there is a critical contraction ratio that separates two distinct vortex breakdown states. Different types of flow modes are distinguished, those of dipole character and those of quadrupole character. These flow modes are related to the level of confinement of the inner vortex core and outer vortex ring. The fundamental effect of outlet contraction is explained through the budget terms in the momentum equations.

The paper is arranged as follows. In the following section the governing equations, in particular the SGS models used in this study are detailed, which is followed by a brief description of the numerical solver and numerical setups used in the study. The results are presented in Section IV, and finally in Section V the main conclusions of this study are given.

\section{Large eddy simulation and its modelling}

\subsection{Governing equations}

The flow velocity considered in this study has a Mach number less than 0.2. For the low Mach number flows considered here the governing equations are approximated by the Navier-Stokes equations for incompressible fluids. In LES these equations are spatially filtered and can be written as,

$$
\begin{gathered}
\frac{\partial \bar{u}_{i}}{\partial x_{i}}=0 \\
\frac{\partial \bar{u}_{j}}{\partial t}+\frac{\partial \bar{u}_{i} \bar{u}_{j}}{\partial x_{i}}=-\frac{1}{\rho} \frac{\partial \bar{p}}{\partial x_{j}}+\nu \frac{\partial^{2} \bar{u}_{j}}{\partial x_{i} \partial x_{i}}-\frac{\partial \tau_{i j}^{r}}{\partial x_{i}}
\end{gathered}
$$

where $u_{i}$ is the velocity component in the Cartesian coordinate $x_{i}$ direction; $p$ and $\rho$ are respectively the static pressure and the density of the gas; $\nu$ is the kinematic viscosity of the mixture. Quantities with overbars are spatially filtered. In the governing equations, unknown terms appear as a result of the spatial filtering. These unknown terms account for the effect of the filtered turbulence scales, hence they are known as the residual stress tensor $\tau_{i j}^{r}$, or sometimes the subgrid scale, SGS, terms. Various SGS models have been developed in the past decades. In this study, to assess the effect of SGS models on the predicted flow three often used SGS models are considered. 


\subsection{Smagorinsky SGS model}

Smagorinsky [22] presented a simple SGS model, based on an analogy to the eddy viscosity model widely used in the RANS simulations.

$$
\tau_{i j}^{r}-\frac{1}{3} \tau_{k k}^{r} \delta_{i j}=-2 \nu_{r} \bar{S}_{i j}
$$

In analogy to the mixing length model of Prandtl, the eddy viscosity is modelled as

$$
\nu_{r}=l_{s}^{2} \bar{S}=\left(C_{s} \Delta\right)^{2} \bar{S},
$$

where $C_{s}$ is the Smagorinsky coefficient; in high Reynolds number turbulent free-shear flows, the value is often taken as 0.15 ; the filter size $\Delta$ is usually taken as the grid cell size; $l_{s}$ is the Smagorinsky length scale. The characteristic filtered rate of strain $\bar{S}$ is

$$
\bar{S}=\left(2 \bar{S}_{i j} \bar{S}_{i j}\right)^{1 / 2}
$$

where $\bar{S}_{i j}$ is

$$
\bar{S}_{i j} \equiv \frac{1}{2}\left(\frac{\partial \bar{u}_{i}}{\partial x_{j}}+\frac{\partial \bar{u}_{j}}{\partial x_{i}}\right)
$$

\subsection{One-equation eddy viscosity SGS model}

An alternative to the Smagorinsky model is the one-equation eddy model (OEE) $[23,24,25]$. The Deardorff one-equation model [23] can be written as,

$$
\nu_{r}=C_{v} k_{s g s}^{1 / 2} \Delta
$$

where $k_{s g s}$ is the turbulent kinetic energy on the sub-grid-scale (residual kinetic energy); the model constant is $C_{v} \approx 0.1$. The residual kinetic energy is obtained from a transport equation, [23]

$$
\frac{\partial k_{s g s}}{\partial t}+\frac{\partial k_{s g s} \bar{u}_{i}}{\partial x_{i}}=\nu_{r} \bar{S}^{2}+\frac{\partial}{\partial x_{i}}\left(\nu_{r} \frac{\partial k_{s g s}}{\partial x_{i}}\right)-C_{\varepsilon} k_{s g s}^{3 / 2} / \Delta,
$$

A typical value of $C_{\varepsilon}$ is 1.048 . 


\subsection{Dynamic SGS models}

In the above two SGS models the model constants are preset as global constants. In the dynamic approach developed by Germano et al. [26] and Lilly [27], the model parameter is determined locally from the resolved scales data. Hence, the model has no free parameter to tune. However, the approach is appropriate only when the behavior of the small scales vary similarly with different filters. Such situation occurs for well resolved turbulent flows away from boundaries. In the present study the Dynamic Smagorinsky and the Dynamic One-Equation Eddy model [28] are considered. The results obtained from these two dynamic SGS models are compared with those obtained from their corresponding non-dynamic version, in order to evaluate the sensitivity of results to the SGS models. The test filter function in the dynamic models

is identical to the base level filter function, and the test filter size $\widehat{\Delta}$ is twice the base level filter size $\Delta$.

\subsection{Numerical methods}

The above governing equations are numerically solved using an open source solver, based on the OpenFOAM library [29]. The spatial discretization is done using a so-called filtered linear scheme, which is essentially a second order central difference scheme. The time integration of the governing equation is done using a second order backward Euler scheme. The swirler, the combustion chamber and the exhaust pipe are described using an unstructured grid system discussed in more details in the next section.

\section{Case set-up and geometry description}

\subsection{Case set-up}

We consider the experimental case of Orbay et al. [3] as the baseline case. The geometry of the experimental rig (which is also the boundary of the computational domain in this study) is shown in Figure 1. The swirler is made up of 15 channels where the air is supplied forming a tangential flow motion when entering into the main chamber. The main chamber has a square cross-section with a width of $2 H=100 \mathrm{~mm}$ and a length of $L=100 \mathrm{~mm}$. On top of the main chamber a cylindrical exhaust pipe is implemented. The diameter of the exhaust pipe is $2 R_{0}=65 \mathrm{~mm}$ and the length is $100 \mathrm{~mm}$. The contraction ratio $C_{r}=R_{0} / H$ is 0.65 . The contraction ratio is defined following Escudier et al. [2,9]. Alternatively, one can define the contraction ratio 


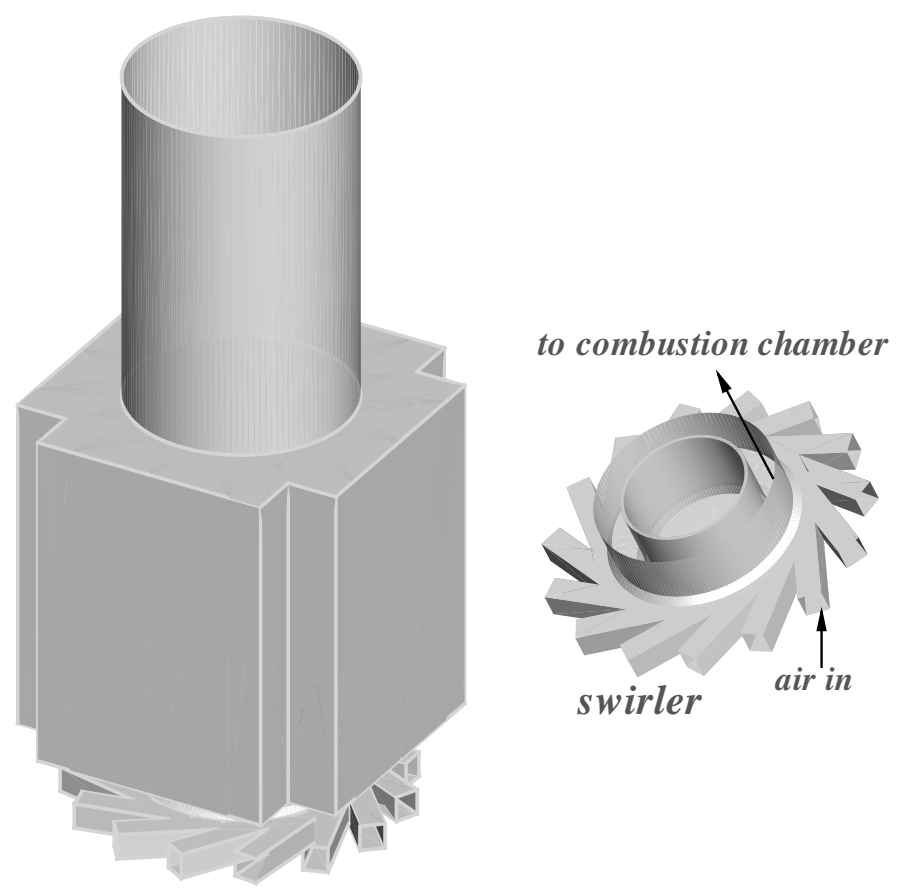

Figure 1: Geometry of the experimental rig; (left) from bottom to top: swirler, main chamber and exhaust pipe; (right) 3-D swirler with 15 inlets.

as the ratio of the cross-section area of the exhaust pipe to the cross-section area of the main chamber. For $C_{r}=0.65$ the area based contraction ratio is 0.365. Note that when computing the cross section area of the main chamber the area taken by the supporting frame at the four corners of the chamber has to be subtracted (cf. Fig.1 of Orbay et al. [3] for details of the geometry). Figure 2 shows the dimensions of the main chamber, the exhaust pipe and the swirler outlet. At the bottom of the main chamber there is a cylindrical bowl. The radius of the bowl is $R_{1}=19 \mathrm{~mm}$ and the length of the bowl is $L_{1}=25 \mathrm{~mm}$. LDV and particle image velocimetry (PIV) measurements were reported for the five upstream axial positions in the symmetric plane indicated by the dashed lines in Figure 2. In the LDV and PIV experiments, the air is preheated to $670 \mathrm{~K}$. The walls are assumed to be adiabatic, hence, the flow is considered to be isothermal [3]. This experimental data is used to validate the LES results. More details of the experimental setup and data uncertainties are referred to the paper of Orbay et al. [3].

Table 1 lists the experimental and simulation conditions. The baseline 


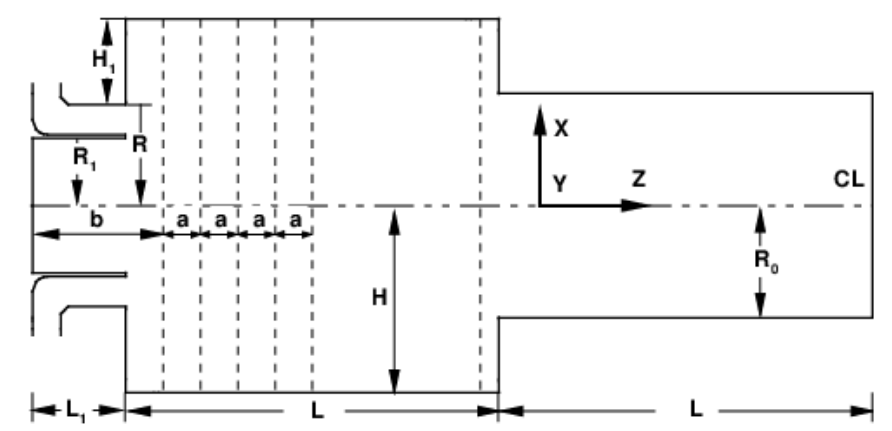

Figure 2: Two dimensional cross-section of the rig with LDV/PIV measurement locations indicated using dashed lines. All dimensions in $\mathrm{mm} ; \mathrm{L}_{1}=25, \mathrm{~L}=100, \mathrm{R}_{0}=32.5, \mathrm{R}_{1}=19$, $\mathrm{R}=27, \mathrm{H}_{1}=23, \mathrm{H}=50, \mathrm{a}=10, \mathrm{~b}=35$.

case where experiments were conducted [3] is the case with a contraction ratio $C_{r}=0.65$. In order to elucidate the effect of contraction on the vortex breakdown and precessing vortex core (PVC), LES is carried out for eight other cases with various contraction ratios, ranging from 0.325 to 1 . In the case with the contraction ratio of one there is no exhaust pipe implemented. Instead, the main chamber is extended to have a total length of $2 \mathrm{~L}=200 \mathrm{~mm}$. The area based contraction ratio varies from 1 to 11 .

In the experiments it is not possible to determine the swirl number at the swirler exit, since the metal swirler prevents optical access for the LDV/PIV measurements. In a previous LES study it was estimated that the swirl number at the swirler exit plane was about 1.4, based on the experimental mean velocity profile slightly downstream the burner exit plane [3]. In the present study the swirler is resolved together with the main chamber and the exhaust pipe. The LES results show that the swirl number at the swirler exit plane is indeed 1.4, based on the time averaged mean velocity field using the definition of swirl number given in Eq. 5. There are different definitions of swirl number or swirl intensity; the most often used one, c.f. Beer and Chigier [30], is defined as the ratio of axial flux of angular momentum to the axial flux of the axial momentum,

$$
S=\frac{M_{\text {angular }}}{R M_{\text {axial }}}=\frac{\int_{0}^{R} U_{\text {axial }} U_{\text {tangential }} r^{2} d r}{R \int_{0}^{R} U_{\text {axial }}^{2} r d r},
$$

where $U_{\text {tangential }}$ is the characteristic tangential velocity, $U_{\text {axial }}$ is axial velocity, $M_{\text {angular }}$ is axial flux of angular momentum, and $M_{\text {axial }}$ is axial flux of 
axial momentum.

The bulk flow velocity at the swirler inlet (area averaged mean velocity $U_{\text {in }}$ ) is $43.5 \mathrm{~m} / \mathrm{s}$, which yields an area averaged mean axial velocity at the swirler exit plane $\left(U_{0}\right)$ (the combustion chamber inlet velocity) approximately $30 \mathrm{~m} / \mathrm{s}$. The area averaged mean flow velocity in the exhaust pipe for the various cases $\left(U_{\text {out }}\right)$ ranges from $41.8 \mathrm{~m} / \mathrm{s}$ (with $C_{r}=0.325$ ) to $3.81 \mathrm{~m} / \mathrm{s}$ (with $C_{r}=1$ ). Based on the mean flow velocity and shear layer thickness at the first experimental plane the Reynolds number is about 20,000. Based on the LDV data at the same plane it is estimated that the integral velocity fluctuation scale is $7 \mathrm{~m} / \mathrm{s}$, the integral length scale $25 \mathrm{~mm}$, and the turbulent Reynolds number $R e_{T}=2600$. Based on Kolmogorov's hypotheses, assuming that $\lambda / l_{0} \approx R e_{T}^{-3 / 4}$ and $\eta / l_{0} \approx R e_{T}^{-1 / 2}$ the Taylor micro-length is $\eta \approx 0.5 \mathrm{~mm}$, while the Kolmogorov length scale is $\lambda \approx 0.07 \mathrm{~mm}$.

\subsection{Computational cases and grid resolution}

For the nine cases with different contraction ratios, after the sensitivity tests for the baseline case (with $C_{r}=0.65$ ), the grid resolution is fixed for all cases. Table 1 shows the details of the grid cell sizes and total number of grid cells for the different contraction ratios. Non-uniform unstructured cells are generated using the snappyHexMesh utility with the maximum aspect ratio of 4.01 and minimum face area of $4.61 \times 10^{-8} \mathrm{~m}^{2}$. The time step is $\Delta t=2.5 \times 10^{-6} s$, the maximum Courant number and mean Courant number are about 0.59 and 0.03 , respectively. For the grid sensitivity test, local refinement is carried out for the baseline case $\left(C_{r}=0.65\right)$, with the cell sizes in the swirler halved in all directions. We opted to refine $100 \%$ the swirler region since it is expected that this is the most crucial part of the geometry for the development of the flow. Refining twice in each direction the full mesh would have lead to too exhaustive requirements on the computer resources. The grid in the main chamber and the exhaust pipe are kept the same as the baseline grid shown in Table 1 . The total cell number increased from 2.28 million to 3.79 million. In the local refinement not only the hexahedral cells, but also the polyhedral cells are employed. The maximum aspect ratio is 3.56 with the minimum face area $3.00 \times 10^{-8} \mathrm{~m}^{2}$. The time step $\Delta t=1 \times 10^{-6} \mathrm{~s}$. The mean Courant number is about 0.015 and the maximum Courant number is about 0.5 .

Parallel computing is performed to reduce the computational time for all the cases. Due to the relatively low number of grid cells required, the computational domain is decomposed into 64 blocks, which involves 64 processors. 


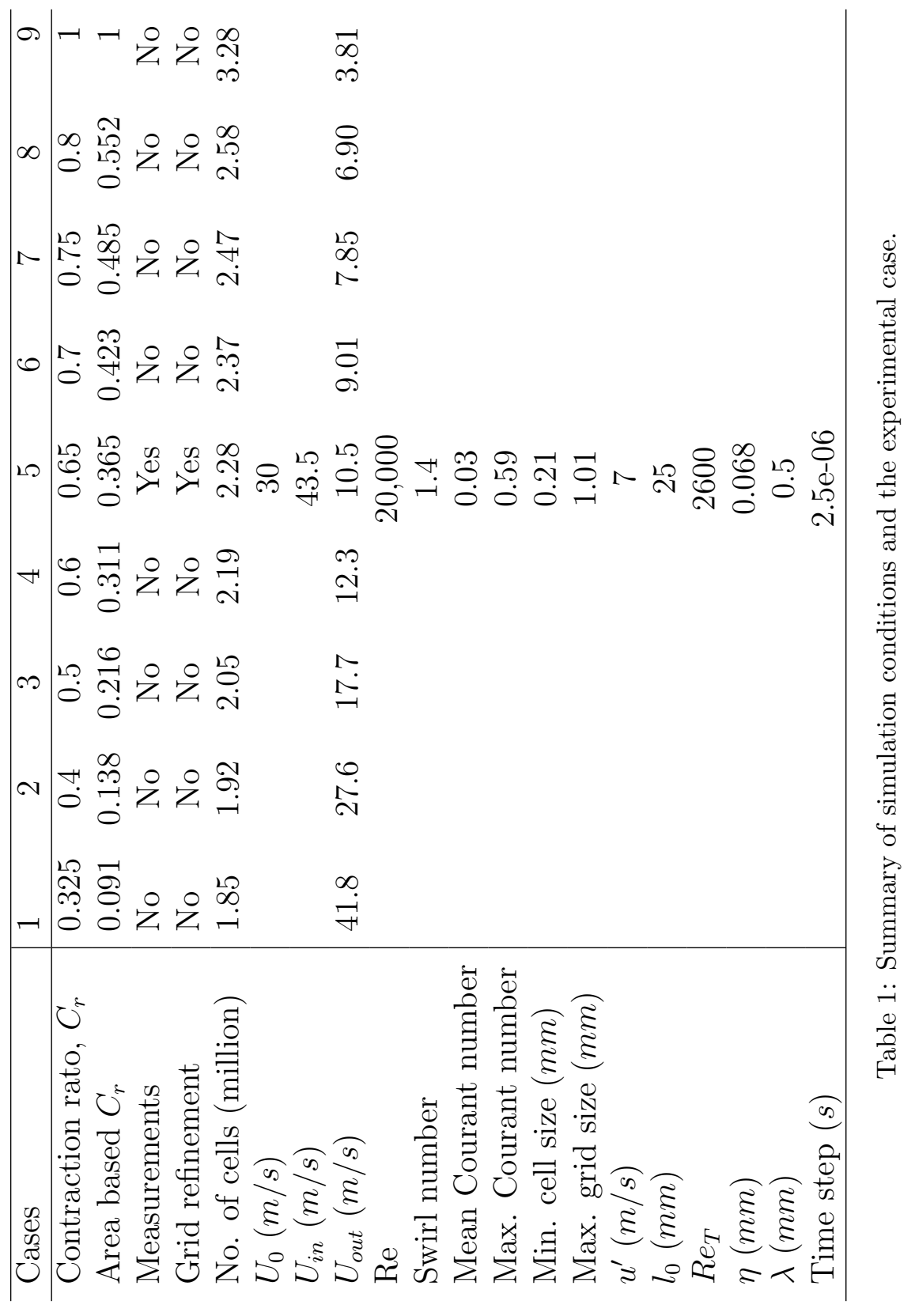


For post-processing purposes, the full 3D field was saved with a frequency of $1 \mathrm{~ms}$ physical time (400 time steps on the base grid resolution, 1000 time steps for the refined case). Although the flow was found to be fully developed around $0.1 \mathrm{~s}$ (15 flow-through times for most cases), the initial computations were carried out for $0.2 \mathrm{~s}$ physical time to assure fully developed conditions. After this initial stage of the simulations data were collected for $400 \mathrm{~ms}$ (ca. 60 flow-through times) for statistical analyses.

\subsection{Initial and boundary conditions}

At the air inlets to the swirler, Dirichlet and Neumann conditions are specified for velocity and pressure, respectively. The normal velocity component is assumed to have a top-hat profile, which is $U_{i n}$ in Table 1 . No turbulence fluctuations are assumed at the inlets. Due to the additional component of shear and the presence of centrifugal instabilities, swirling flows are characterized by a higher rate of turbulence generation (compared with their non-swirling counterparts); thus, the omission of the inlet turbulence is expected to have negligible effects on the flow.

A no-slip condition is assumed on all walls, along with a zero-gradient condition in the normal direction for the pressure. To allow for the use of relatively coarse grid resolution near the wall, wall functions [24, 31, 32, 33] are adopted for evaluating the SGS turbulent kinetic energy and velocity at the first grid near the wall. At the outlet, a zero gradient condition is applied for the flow variables, with the total pressure and a flux corrected velocity specified for the pressure and velocity, respectively. The initial pressure is constant and equal to the pressure at the swirler inlet, which is $1 \mathrm{~atm}$. Since the flow studied here is statistically stationary, the results are independent of the initial field. The initial velocity field for the baseline case $\left(C_{r}=\right.$ $0.65)$ is simply assumed to be zero. For other cases the initial velocity and pressure fields are mapped from an instantaneous field of the baseline case. For each case convergence of the statistical mean fields and the corresponding variances is monitored to ensure the statistical results are independent of the initial fields.

\section{Results and discussion}

First, the accuracy of the LES results is examined based on the baseline case by comparing the LES results with experimental data and by examining the sensitivity of the LES results to SGS models and grid resolution. Then, 


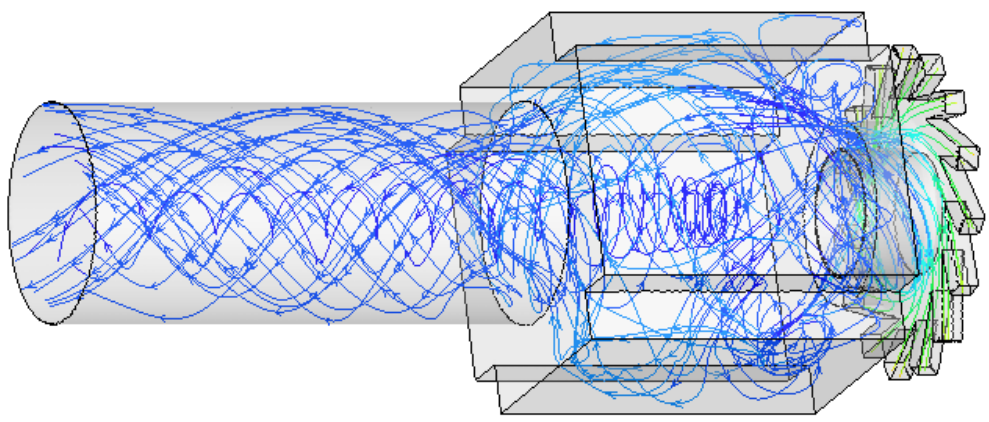

Figure 3: 3D view of the mean flow streamlines in the baseline case $\left(C_{r}=0.65\right)$.

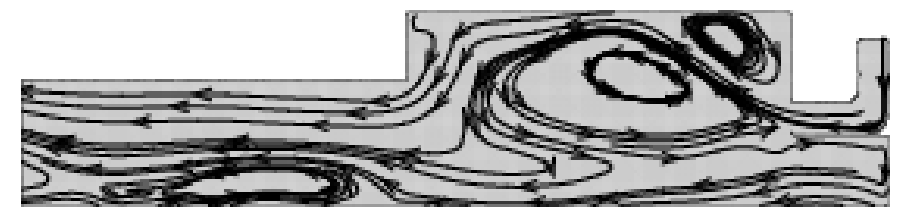

Figure 4: 2D mean flow streamlines in the symmetric plane of the baseline case $\left(C_{r}=0.65\right)$. Only half of the geometry is shown.

the effect of the outlet contraction on the vortex breakdown and PVC is discussed. The mean flow field shows different vortex breakdown structures when the contraction ratio is changed from 0.325 to 1.0. In particular, along the axis of the chamber the flow reversal is shown to change drastically when the contraction ratio is increased. The underlying flow physics is analyzed by comparing the budget terms in the momentum equations. Finally, the unsteady large-scale structures in the flows with different outlet contractions are explored.

\subsection{The mean flow field}

We begin with examining the LES results obtained using the baseline grid without local refinement in the swirler, and the one-equation eddy (OEE) SGS model. First, the mean flow structure from LES for the baseline case is displayed in Figures 3 and 4 where the key flow characteristic can be identified. The 3D streamlines show a complex flow structure in the swirler, the main chamber and the exhaust pipe, with different 3D rotational structures along various directions. Focusing on the 2D view of the streamlines in the symmetric plane of the geometry (Figure 4) one can see that in the 

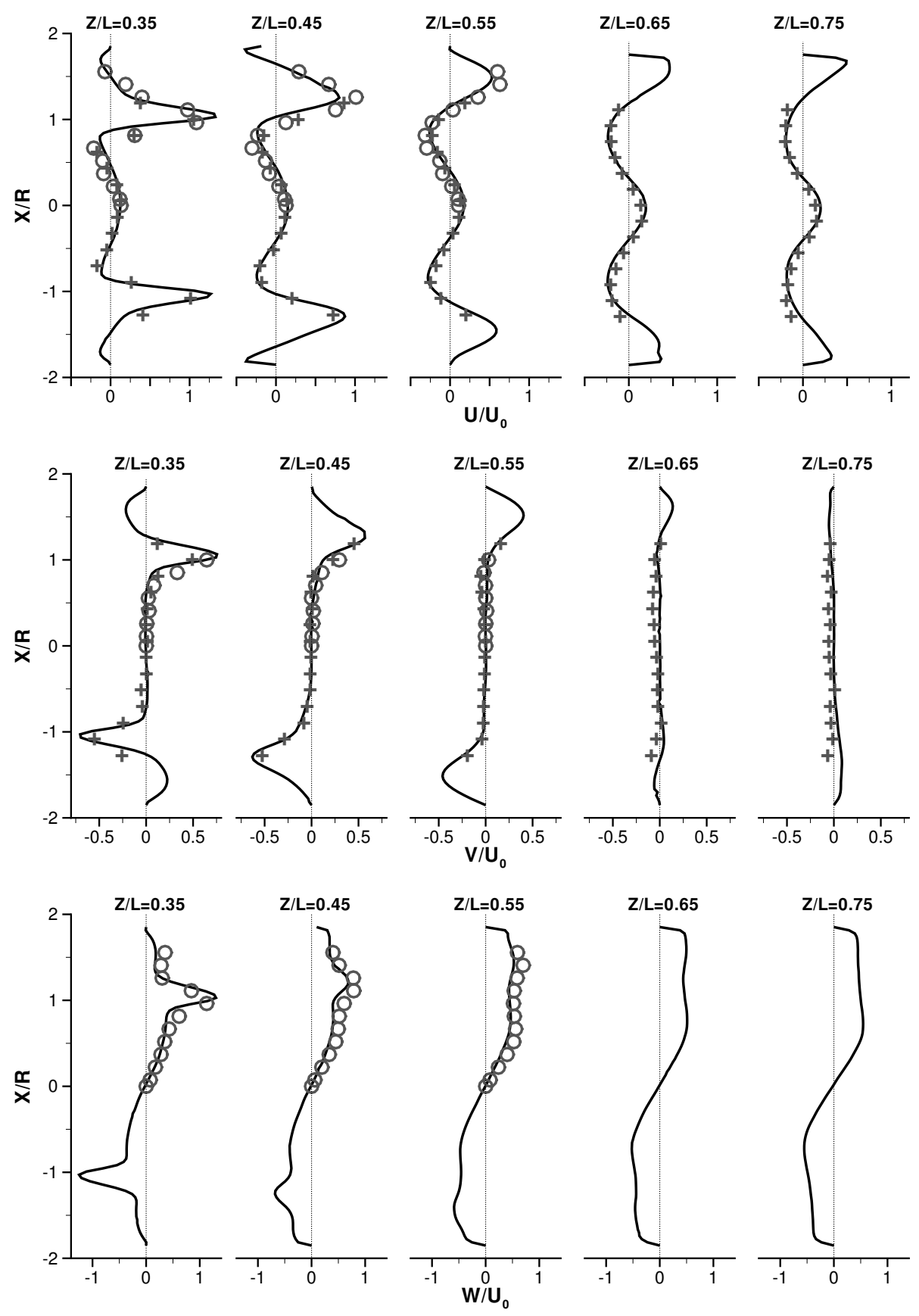

Figure 5: Mean velocity components along the radial direction $(X)$ at different axial positions $(Z)$ for the baseline case $\left(\mathrm{C}_{r}=0.65\right)$; LES $(-)$, PIV $(+)$, LDV (o). 

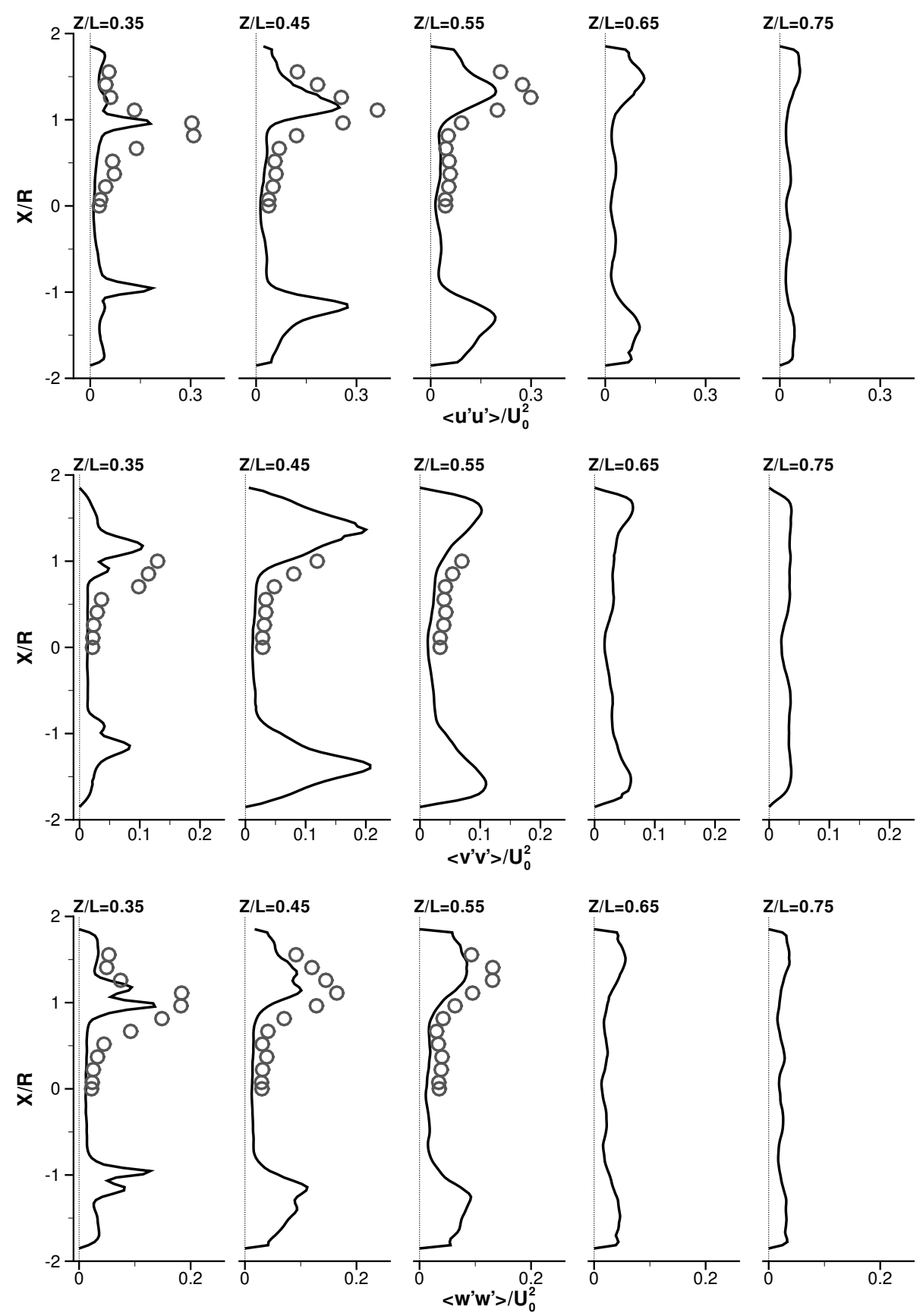

Figure 6: Reynolds stresses along radial direction $(X)$ at different axial positions $(Z)$ for the baseline case $\left(\mathrm{C}_{r}=0.65\right)$; LES (-), LDV (o). 
main chamber there are three recirculation zones formed. Near the upstream corner a corner recirculation zone (CRZ) is formed owing to the sudden expansion. Next to the CRZ there is a large recirculation zone, which is referred here to as the central toroidal recirculation zone (CTRZ). This recirculation zone is a result of the vortex breakdown under the present high swirl condition. Near the axis of the main chamber is the third recirculation zone, which is a secondary vortex breakdown due to the exhaust pipe contraction. As one can observe in Figure 4, multiple vortex breakdowns are generated in the flowfield. Such multiple (up to four) breakdowns have been observed previously, e.g., by Sarpkaya [34]. The vortex breakdowns observed here are of bubble type, cf. Billant, Chomaz, and Huerre [35] for a review of different vortex breakdown types.

The LES results are compared with the experiments for the baseline case. In the figures to be discussed below the mean velocity has been normalized with the velocity $U_{0}=30 \mathrm{~m} / \mathrm{s}$. The Reynolds stresses are normalized using $U_{0}^{2}$. The radial distance is normalized with $R$ whereas the axial distance with the combustion chamber length, $L$, (see Figure 2 for the definition of the lengths). As seen in Figure 5, the LES results are in good agreement with the PIV and the LDV data for the three mean velocity components. At $Z / L=0.35$, in the near wall region (with $X / R$ larger than 1.5 ), the axial velocity component is less than zero. Negative axial velocity means that the line at $Z / L=0.35$ is crossing the corner recirculation zone. The same phenomena can be observed at $Z / L=0.45$. At the center line of the main chamber, the axial velocity component has a positive value and the flow direction is from the inlet to outlet for all the axial locations shown in the figure.

As shown in Figure 5, the peak radial velocity is seen in between the CRZ and large CTRZ, which corresponds to the expansion of the flow from the annular swirler exit to the main chamber. Due to the confinement of the wall the radial velocity component becomes zero at the wall. As seen from Figure 4, the line $Z / L=0.35$ crosses the CRZ, leading to the negative axial velocity at $X / R>1.5$ and the negative radial velocity at $X / R>1.25$. It is interesting to note that in the central part of the chamber $X / R<0.75$, the radial velocity is zero, indicating that the flow is parallel to the axis of the chamber.

The tangential velocity agrees also very well with the LDV data (the 2D PIV did not include the tangential component). At $Z / L=0.35$ one can see a peak in the tangential velocity, which decays downstream. Around $X / R=0$ 
(the centerline of the main chamber) the tangential velocity resembles a solid body rotation profile. Outside this center region the tangential velocity is nearly constant along the radial $(X)$ direction.

\subsection{Reynolds stress and turbulence}

Figure 6 shows the evolution of the normalized Reynolds stresses obtained from the LDV measurements and LES. In the center region of the chamber $(X / R<0.5)$, the Reynolds stresses are rather low. This is owing to the low speed parallel flow motion in the region as discussed earlier. The peak of the Reynolds stress is seen in the high speed region where turbulence is produced in the shear layer of the high speed flow stream. The width of the shear layer increases gradually downstream of the swirler exit plane, and as such the gradient of the mean velocity decreases, leading to a lower production rate of turbulence downstream. The Reynolds stresses are seen to decrease along the downstream direction.

As it is common for higher order statistics, the Reynolds stresses from LES are in a less good agreement with the LDV data than the mean velocity profiles. All LDV data show higher Reynolds stresses than the LES results. This discrepancy is partly due to the use of the relatively coarse grid resolution in the swirler and to the used OEE SGS model. The grid sensitivity and SGS model sensitivity studies will be given in the next section.

\subsection{Sensitivity to $S G S$ model and grid resolution}

Two locations, $Z / L=0.35$ and 0.55 , are chosen to examine the sensitivity of the LES results to grid resolution and SGS model. The tested SGS models are the OEE model (which is the baseline model used to obtain the LES results discussed earlier), the Dynamic One-Equation Eddy (DOEE) model, and the dynamic Smagorinsky (DS) model. The results are shown in Figure 7. The mean velocity profiles show a moderate sensitivity to the SGS models and grid resolution. The basic flow structures, hence the vortex breakdown, are well captured for all SGS models.

For the Reynolds stresses the LES results are seen to be sensitive to the SGS model. At the near swirler plane $Z / L=0.35$, the DOEE model gives rise to higher Reynolds normal stresses (which are the components of turbulent kinetic energy), while the DS model results in an even higher turbulence kinetic energy, and a slightly better agreement with the experiments than the other models. At the downstream location $Z / L=0.55$, the OEE model predicted a higher turbulence kinetic energy that is in closer agreement with 

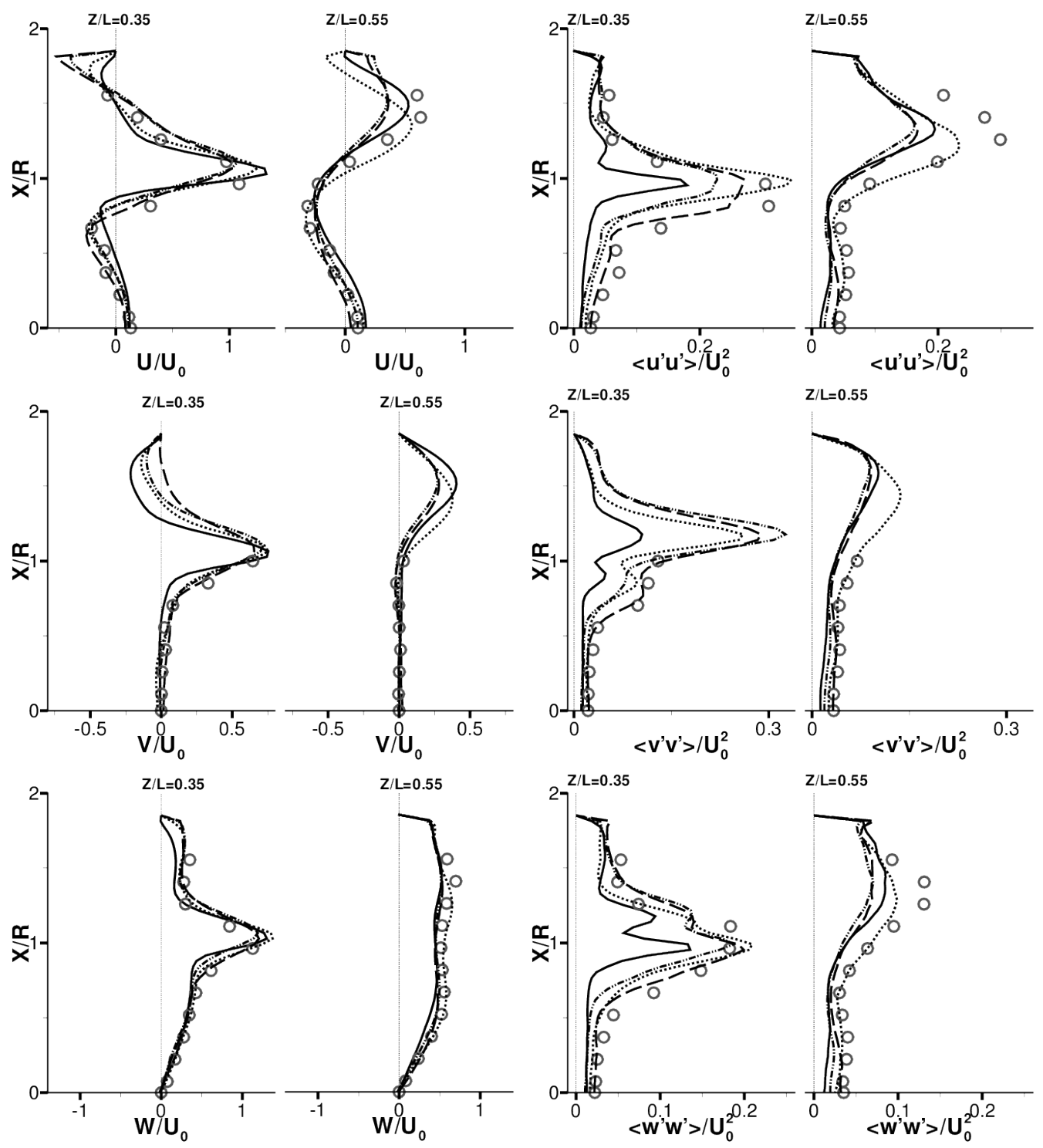

Figure 7: LES results obtained using different SGS models and grid resolution for the baseline case $\left(\mathrm{C}_{r}=0.65\right)$. Left column: the mean velocity components; right column: the Reynolds stress components. - : one equation eddy (OEE) model; -..-: dynamic one equation eddy (DOEE) model; - -: dynamic Smagorinsky (DS); $\cdots$ swirler local grid refinement and OEE; o: LDV. 
the experiments. Therefore it is not conclusive that the dynamic models, which is computationally more time consuming, give a better prediction of the turbulence field. Furthermore, one may notice that the sensitivity of the LES results to SGS models is much lower in the downstream as compared with that in the upstream. For this reason in LES of other cases with different outlet contraction only the OEE model is used.

As seen in Figure 7, the results at the near swirler plane $Z / L=0.35$ are more sensitive to the SGS models than those downstream. This might be partly due to the insufficient grid resolution in the swirler region, which can not resolve the fine detailed flow structures and turbulence production in the swirler. To investigate this, a local grid refinement in the swirler geometry is carried out, in which the cell sizes are halved in all directions in the entire swirler geometry. One can see that with the baseline OEE model but finer grid resolution in the swirler, the prediction of the turbulence kinetic energy is improved in both the upstream and downstream locations.

One may note that the present baseline grid and the OEE model are rather adequate for the prediction of the mean flow field; the results are moderately sensitive to either the SGS models or the grid refinement. The turbulence field is relatively more sensitive to the SGS models and the grid refinement. Since the main goal of the paper is to study the effect of outlet contraction on the flow structures, the baseline grid resolution is used for its moderately low computational demand. This resolution is adequate to capture the main flow features and still affordable to carry out a sensitivity study with a series of different cases simulated.

\subsection{Effect of outlet contraction on the mean flow field}

To understand the effect of outlet contraction on the flow structure, nine cases are simulated using the one equation eddy model and the baseline grid (without local refinement in the swirler). The resulting flow fields are presented in Figure 8, where the mean flow streamlines are displayed for half of the symmetric cross-section. Several observations can be made: first, the corner recirculation zone and the central toroidal recirculation zone (discussed in Section 4.1) are rather similar in all cases. With the exhaust pipe contraction the centers of the CRZ and CTRZ are compressed slightly towards upstream. The largest exhaust pipe contraction effect is on the flow around the axis of the main chamber. Without contraction $\left(C_{r}=1\right)$, the flow stream on the axis is from downstream to upstream, owing to the large CTRZ. With the exhaust pipe contraction, cf. the case with $C_{r}=0.8$, the CTRZ is split 


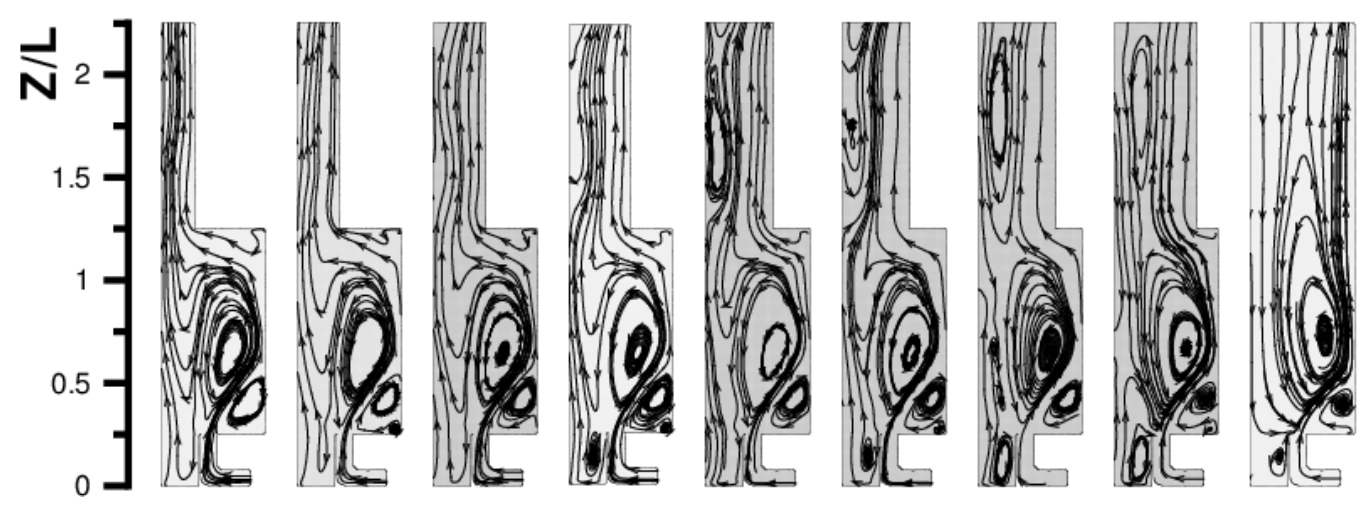

Figure 8: Mean flow field streamlines of nine cases with different contraction ratio; from left to right: $C_{r}=0.325,0.4,0.5,0.6,0.65,0.7,0.75,0.8$ and 1 .

into two recirculation zones: one in the main chamber and one in the exhaust pipe. Further decreasing the diameter of the exhaust pipe, the recirculation zone in the exhaust pipe is compressed away from the pipe and eventually it is pushed away, e.g. when $C_{r}<0.5$. The mean flow in the exhaust pipe is then completely towards the downstream direction. As a result, as the diameter of the exhaust pipe decreases, the flow on the axis of the main chamber changes its direction, from reversed flow at $C_{r}=1$ to flow towards downstream.

Figure 9 shows two radial lines in the main chamber; one is slightly downstream of the swirler exit, $Z / L=0.35$, and the other is slightly upstream of the sudden contraction plane before the exhaust pipe, $Z / L=1.2$. At the near swirler plane $(Z / L=0.35)$ the axial, radial and tangential velocity components in the outer region $(X / R>0.75)$ are rather similar in various cases, corresponding to the observation that the outlet contraction exerted minor impact on the CRZ. In the region close to the main chamber axis $(X / R<0.75)$ the axial and tangential velocity components are rather sensitive to the downstream contraction. In particular, a decreasing diameter of the exhaust pipe leads to an enhanced positive axial velocity and an enhanced tangential velocity. At the downstream location $(Z / L=1.2)$, the outlet contraction has a significant impact on the entire velocity profile. When the contraction ratio is decreased from 1 to 0.325 , a swirling jet-like velocity profile is seen in the center of the chamber $(X / R<0.5)$. This jet-like characteristic can not be found for the case without a contraction $\left(C_{r}=1\right)$. This structure is insensitive to the upstream bowl geometry and the swirler 

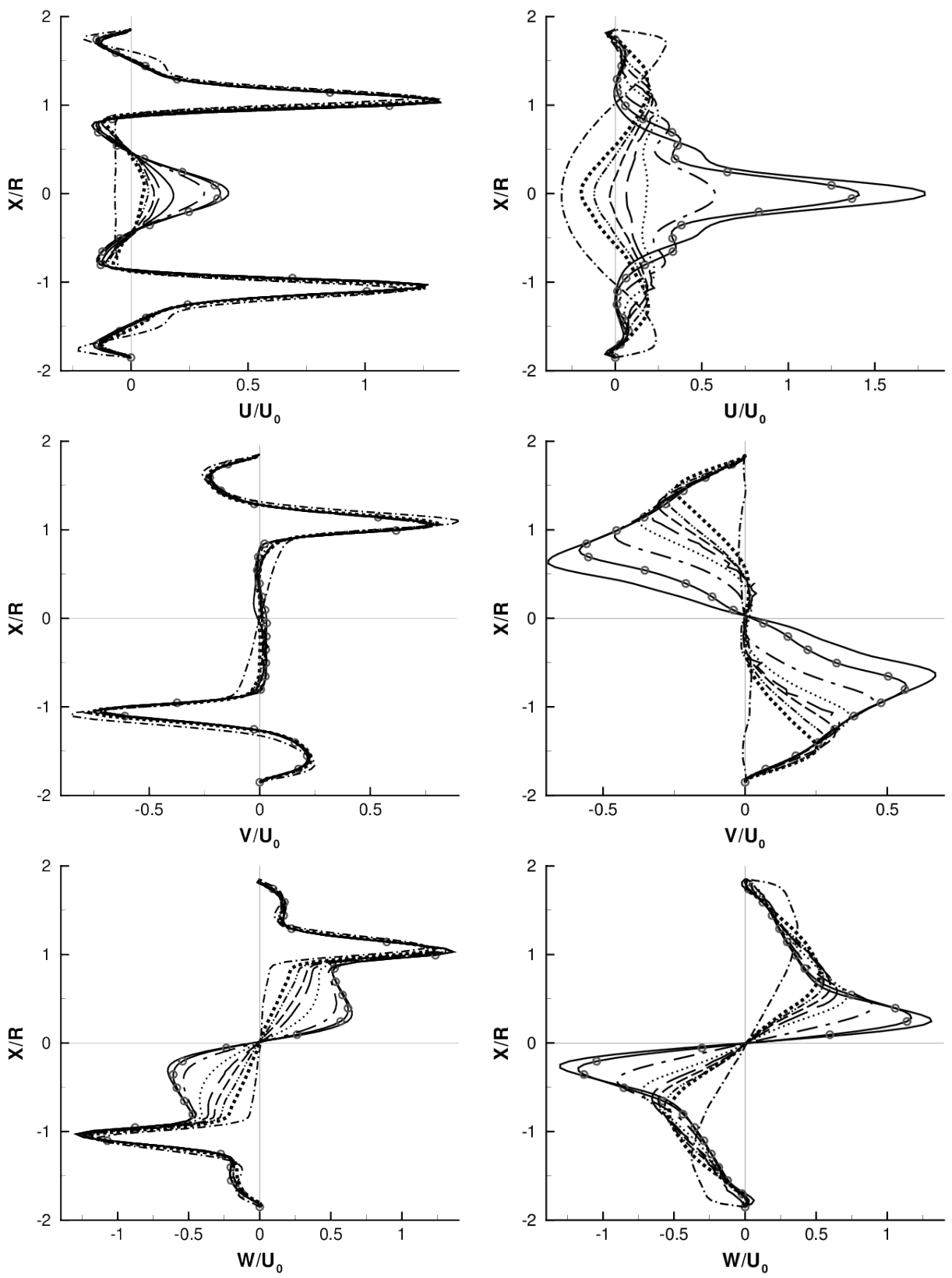

Figure 9: Effect of contraction ratio on the velocity distribution, shown along two lines; left column: $Z / L=0.35$, right column: $Z / L=1.2$. - $: \mathrm{C}_{r}=0.325 ;-\ominus-: \mathrm{C}_{r}=0.4 ;-$ $-: \mathrm{C}_{r}=0.5 ; \cdots: \mathrm{C}_{r}=0.6 ;--: \mathrm{C}_{r}=0.65 ;--: \mathrm{C}_{r}=0.7 ; \cdots \cdot-\cdot \mathrm{C}_{r}=0.75 ; \ldots: \mathrm{C}_{r}=0.8$; $-\cdot-\mathrm{C}_{r}=1$. 

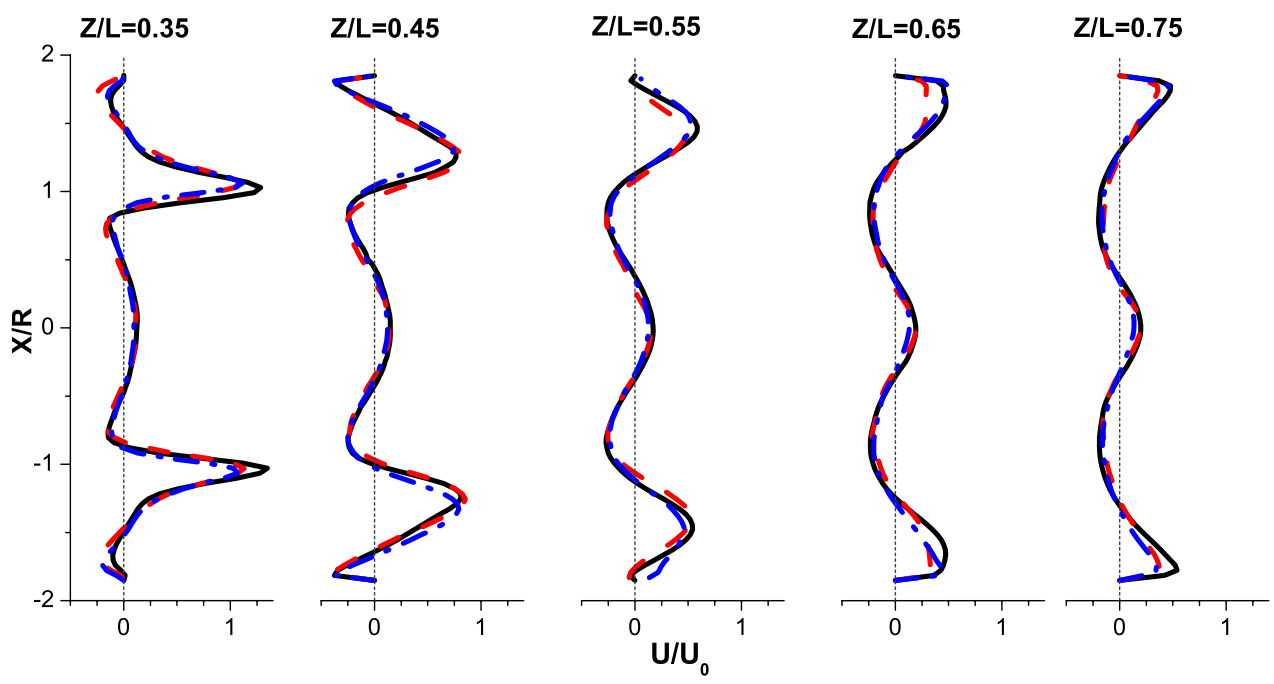

Figure 10: Mean axial velocity component along the radial direction $(X)$ at different axial positions $(Z)$ for the baseline case $\left(\mathrm{C}_{r}=0.65\right)$ from LES with different exhaust pipe lengths; — : $100 \mathrm{~mm},-—: 50 \mathrm{~mm} ;-\cdot-: 150 \mathrm{~mm}$.

exit. Similar effects of the outlet contraction can be observed in the axial velocity profiles reported by Escudier, Bornstein, and Zehnder [2].

For confined swirling flows, two very important swirling flow models are the Rankine vortex [36] and the Q vortex [37]. The Rankine vortex assumes that the tangential velocity is a linear function of the distance to the axis of the pipe in the center region, followed by an inverse function of the radial distance to the axis in the near wall region. One can see that the tangential velocity in the downstream location $Z / L=1.2$ is similar to the Rankine vortex. Figure 9 shows a solid body rotation in the center part. The gradient of the tangential velocity is different for different contraction ratios. The lower the contraction ratio is, the higher the tangential velocity is, and the smaller the core of the solid body rotation. This is owing to the conservation of angular momentum: with smaller rotation core (confined by the exhaust pipe) the tangential velocity is enhanced.

As shown in Figure 8 when $C_{r}>0.6$ there is a recirculation region at the outlet plane. Since a zero-velocity-gradient condition has been used as the outflow condition one may suspect that the recirculating flow at the outlet plane may affect the flow field upstream in the main chamber. If the flow is at high Mach number with significant compression effect the problem may 
be ill-posed. In the present case the flow is at a low Mach number and incompressible condition, thus the problem is well-posed when a global mass conservation is enforced to determine the mass flow rate at the outlet. The existence of recirculation region at the outlet may nevertheless affect the flow field upstream and in certain cases the statistically mean recirculation region may become asymmetric with the outgoing flow along one (randomly chosen) side of the wall and the inflow on the opposite side of the wall, a phenomenon known as the Coanda effect. To examine whether the Coanda effect is important or whether the recirculation region at the outlet has an important impact on the flow in the main chamber we have performed simulations with different length of the exhaust pipe. Figure 10 shows that the mean flow field in the main chamber is insensitive to the length of the exhaust pipe. In the experiments the length of the exhaust pipe is about $300 \mathrm{~mm}$. The fact that the experimental results agree well with the numerical results computed with different lengths of the exhaust pipe indicates that the Coanda effect is not significant in the present case.

Numerical simulations have been carried out for the $C_{r}=0.65$ and $C_{r}=1$ cases with a large open domain of the size $15 H \times 15 H$ (where $H$ is the half width of the main chamber, cf. Figure 2). Vortex breakdown was shown to occur when the flow from the exhaust pipe was discharged to the open domain. However, the flow structures in the main chamber was shown to be insensitive to the flow field in the downstream open domain (for brevity the results with the open downstream domain are not shown here).

\subsection{The role of pressure gradient and centrifugal force}

One of the vortex breakdown theories is in analogy to separation of twodimensional boundary layers [38]. According to this theory, the quasi-cylindrical and boundary-layer approximations are used to explain why a vortex core exists and what it looks like in a swirling flow system. For the analysis of the development of the flow, some assumptions are required, i.e., the flow is steady, laminar, incompressible and axisymmetric. The momentum equation in cylindrical coordinates along the radial direction can be simplified as:

$$
\frac{w^{2}}{r} \approx \frac{\partial p}{\partial r}
$$

Here, $r$ is the radial coordinate, $p$ is the pressure and $w$ is the tangential velocity. The left hand side and right hand side of Equation (6) respectively 
represent the centrifugal force and pressure gradient. Equation (6) was developed for laminar flows. For turbulent flows other terms, e.g., Reynolds stresses, are shown to play an important role in certain flow configurations, e.g., swirling jet flows, cf. Facciolo et al. [39]

It appears that Equation (6) is valid in the present turbulent flows. In order to evaluate the balance of the different terms in the radial momentum equation, the different terms in the equation of the radial momentum are examined. The dominant terms are the pressure gradient and centrifugal force which are of the same order of magnitude. All other terms, the convection, diffusion and Reynolds stress terms are relatively small so they can be neglected.

The effect of the centrifugal force can be observed from the pressure distribution along the radial direction. Figure 11 shows that the pressure gradient is larger in the center region $(X<R)$ than in the outer region $(X>R)$. The gradient of pressure in the radial direction is enhanced by the decrease of the diameter of the exhaust pipe. From Equation (6) and the tangential velocity profiles shown in Figure 9, it is clear that the change of the pressure distribution in the flow field is owing to the effect of the centrifugal force. Owing to the change of the radial gradient of pressure the axial pressure gradient is affected, which in turns affects the vortex breakdown structures.

Hall [38] was the first to demonstrate that the axial pressure gradient consists of two components, the far field external pressure gradient plus the swirl contribution. Integrating Equation (6) along the radial direction and computing the derivatives along the axial direction, one has:

$$
\begin{gathered}
\frac{\partial}{\partial z} \int_{0}^{R} \frac{\partial p}{\partial r} d r \approx \frac{\partial}{\partial z} \int_{0}^{R} \frac{w^{2}}{r} d r \\
\left(\frac{\partial p}{\partial z}\right)_{r=R}-\left(\frac{\partial p}{\partial z}\right)_{r=0} \approx \int_{0}^{R} \frac{\partial}{\partial z}\left(\frac{w^{2}}{r}\right) d r
\end{gathered}
$$

The physical meaning of the right hand side of Equation (8) is the intensity of the vortex core rotation. The right hand side can be rewritten as Hall [38]:

$$
\int_{0}^{R} \frac{\partial}{\partial z}\left(\frac{w^{2}}{r}\right) d r \equiv \alpha \Gamma^{2} / R_{c}^{3}
$$

where $\alpha$ is the order of magnitude of the divergence of the stream $U_{\text {radial }} / U_{\text {axial }}$, $\Gamma$ and $R_{c}$ are the order of magnitude of circulation and the radius of the vortex core, respectively. From Figure 11, the radius of the vortex core can be 

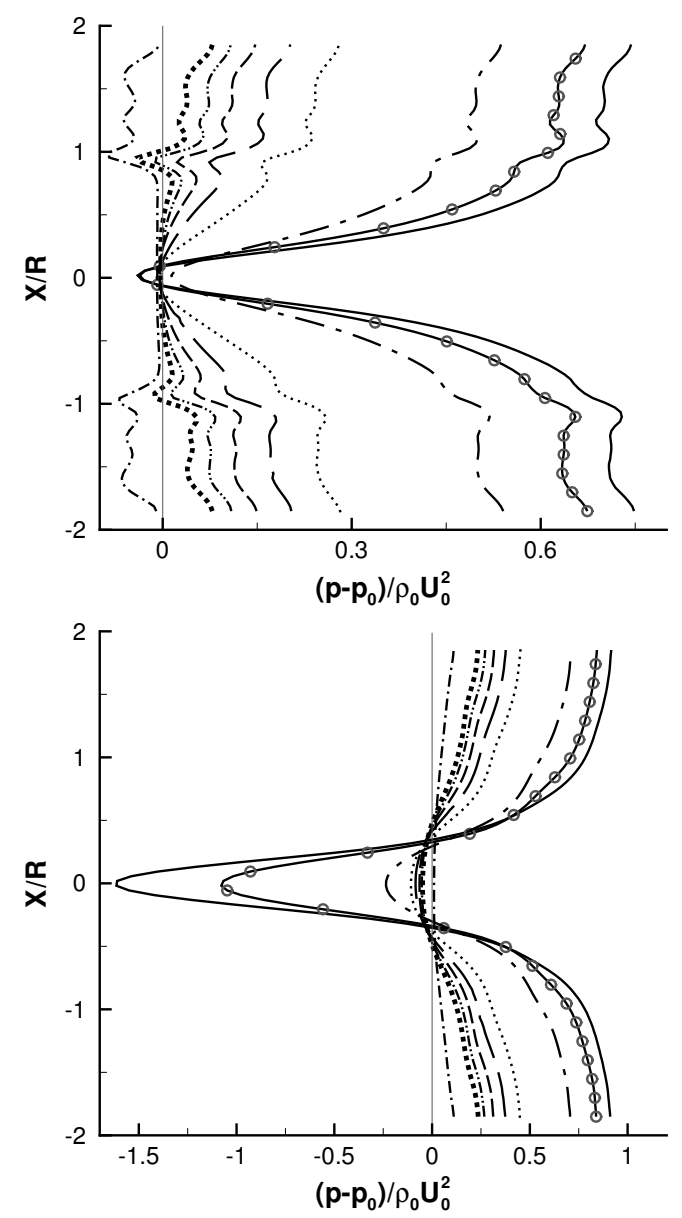

Figure 11: The pressure distribution along radial lines at $Z / L=0.35$ (left) and $Z / L=1.2$ (right) cross section. - - $\mathrm{C}_{r}=0.325 ;-\ominus-\mathrm{C}_{r}=0.4 ;--: \mathrm{C}_{r}=0.5 ; \cdots: \mathrm{C}_{r}=0.6 ;-$ $-: \mathrm{C}_{r}=0.65 ;--: \mathrm{C}_{r}=0.7 ;-. .-: \mathrm{C}_{r}=0.75 ; \ldots .: \mathrm{C}_{r}=0.8 ;-\cdot-: \mathrm{C}_{r}=1$. 
estimated as $R_{c} \approx R$ (which is the radius of the swirler exit). For the nine contraction ratio cases discussed earlier, the radius of the core is the same because these cases have the same swirler and the same main chamber.

The left hand side of Equation (8) is the difference between the axial pressure gradient across the vortex core. From Figure 11, compared with the axial pressure gradient along the center axis, the axial gradient of pressure along the outer edge of the vortex is very small,

$$
\left(\frac{\partial p}{\partial z}\right)_{r=R} \approx 0
$$

Substituting Equation (9) and (10) into Equation (8), one has:

$$
\left(\frac{\partial p}{\partial z}\right)_{r=0} \approx-\alpha \Gamma^{2} / R_{c}^{3}
$$

From Equation (11), it is clear that the axial pressure gradient along the chamber axis is proportional to the swirl number. With decreasing diameter of the exhaust pipe, the tangential velocity (the swirl velocity) is enhanced, and thus the axial pressure gradient is enhanced. Figure 12 shows the axial pressure distribution along the axis of the main chamber for the nine different $\mathrm{C}_{r}$ cases. Since the right hand side of Equation (11) is negative the pressure decreases along the axial direction downstream. This drives the flow on the chamber axis to flow towards downstream of the main chamber, which explains the vortex breakdown structure observed earlier in Figure 8.

Figure 12 shows that the location of the lowest pressure on the axis of the main chamber moves downstream towards the contraction plane as the diameter of the exhaust pipe decreases. Downstream of the contraction plane, the flow in the exhaust pipe experiences a certain recovery in pressure, which corresponds well with the axial velocity distribution along the axis of the main chamber as shown in Figure 13. The minimum of pressure corresponds to the maximum of axial velocity. For the cases with $C_{r} \geq 0.65$, the axial velocity on the center axis changes sign (from positive to negative) indicating a stagnation point on the axis. For the cases with $C_{r} \leq 0.5$, the axial velocity on the centerline is positive indicating a flow along the downstream direction throughout the domain and no stagnation point on the centerline of the chamber. The case $C_{r}=0.6$ is a critical case in which the vortex breakdown changes its structure from one type to another.

To examine if there is a hysteresis effect when varying the contraction ratio around $C_{r}=0.6$, simulations have been carried out with both successive 

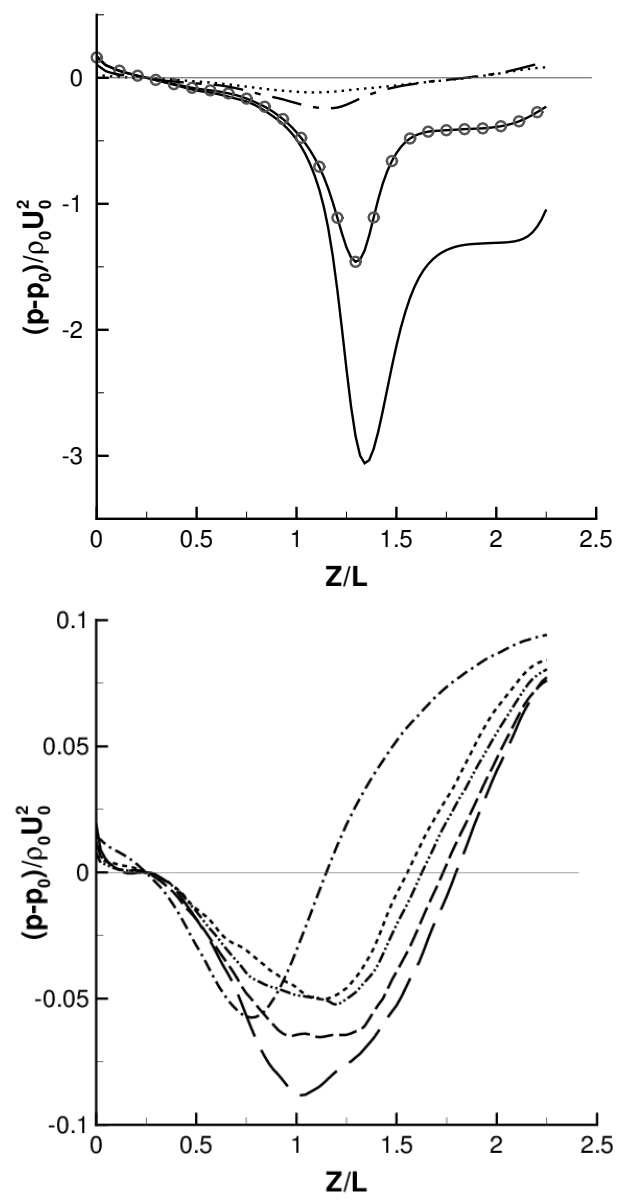

Figure 12: Pressure distribution along the center line for the nine cases. - $: \mathrm{C}_{r}=0.325$; $-\ominus-: \mathrm{C}_{r}=0.4 ;--: \mathrm{C}_{r}=0.5 ; \cdots: \mathrm{C}_{r}=0.6 ;-\longrightarrow: \mathrm{C}_{r}=0.65 ;--: \mathrm{C}_{r}=0.7 ;-\cdot \cdot-: \mathrm{C}_{r}$ $=0.75 ; \ldots . \mathrm{C}_{r}=0.8 ;-\cdot-: \mathrm{C}_{r}=1$. 


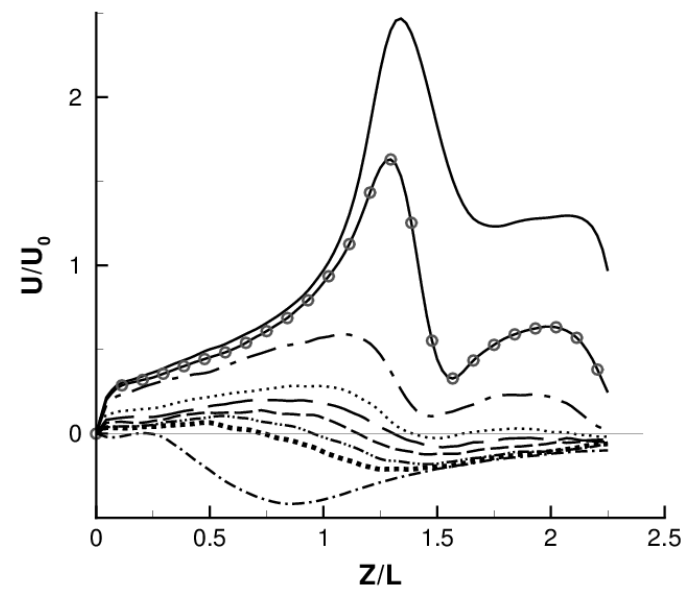

Figure 13: Mean axial velocity on the center axis of the main chamber. - : $\mathrm{C}_{r}=0.325$; $-\ominus-: \mathrm{C}_{r}=0.4 ;--: \mathrm{C}_{r}=0.5 ; \cdots: \mathrm{C}_{r}=0.6 ;-\longrightarrow: \mathrm{C}_{r}=0.65 ;--: \mathrm{C}_{r}=0.7 ;-\cdot \cdot-: \mathrm{C}_{r}$ $=0.75 ; \ldots . \mathrm{C}_{r}=0.8 ;-\cdot-: \mathrm{C}_{r}=1$.

decreasing and successive increasing of $C_{r}$. It appears that the mean flow structures at $C_{r}=0.6$ are independent of the initial state of the simulations. Thus, no hysteresis effect is detected.

To understand the reason why the pressure and axial velocity reach its maximum/minimum at the same location (cf. Figure 12 and Figure 13), one may consider the momentum equation along the axial direction. The leading order terms in the axial momentum equation are the convection term and the axial pressure gradient,

$$
\frac{\partial u^{2}}{\partial z} \approx-\frac{\partial p}{\partial z}
$$

These axial gradients become zero at the same location, thus reaching their maximum/minimum at the same location. This is true for all $\mathrm{C}_{r}$ cases.

4.6. Effect of outlet contraction on unsteady large scale rotational structures

Based on the tangential velocity profiles in Figure 9, the flow is divided into an inner vortex core and an outer vortex ring. The relative strength of these two rotational regions vary with the contraction ratio. Figure 14 shows instantaneous pressure fields in a symmetry plane along the axis of the main chamber for the nine cases with different exhaust pipe diameters, together with corresponding 3-D tracelines. The pressure fields show that the inner vortex core has a relatively low pressure, a feature which is enhanced as the 


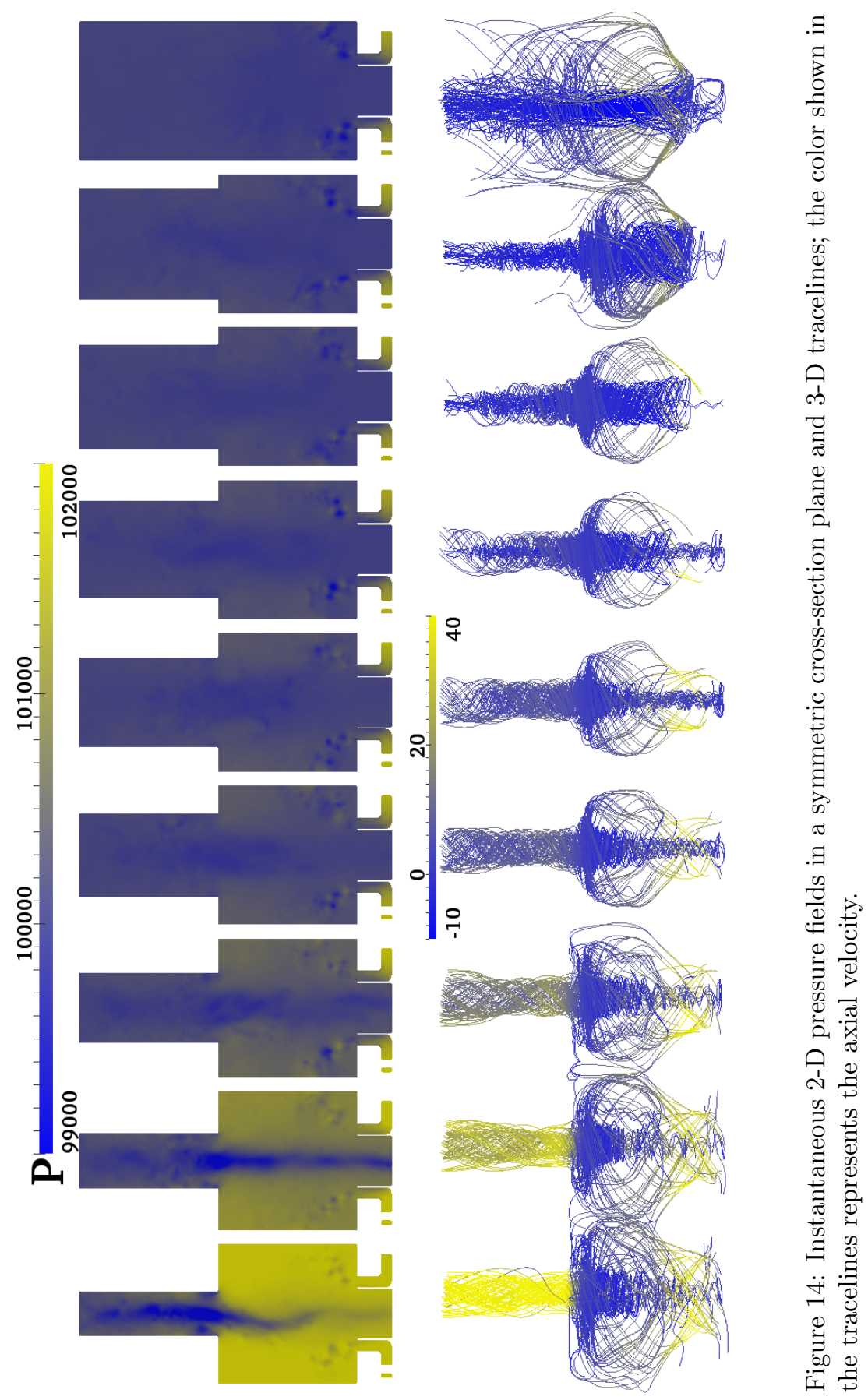



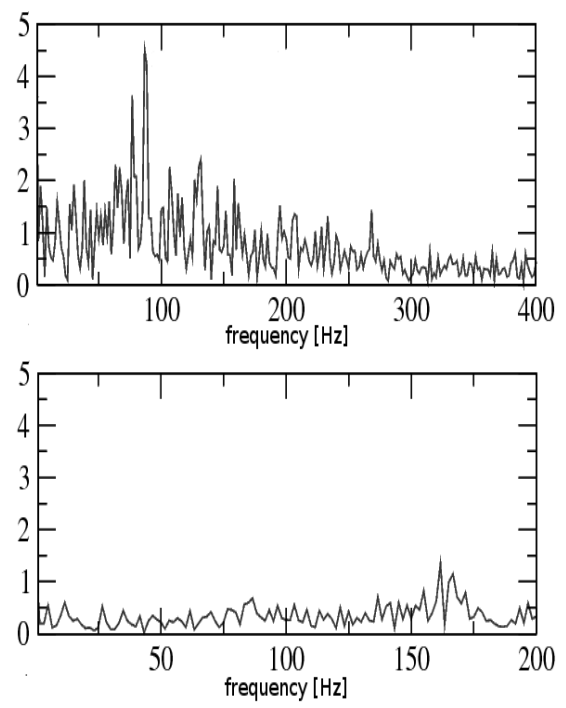

Figure 15: Power spectral densities for the case with the smallest exhaust pipe diameter. The spectra are derived from radial velocity components taken from points at the axial position $Z / L=0.75$. The top and bottom spectra come from the center axis $(X / R=0)$ and an off-center location $(X / R=1.84)$, respectively.

diameter of the exhaust pipe decreases. Additionally, for the case with the smallest diameter $\left(C_{r}=0.325\right)$, a highly twisted vortex core is seen.

To identify the precessing vortex core (PVC) motion, along with other structures in the flow, spectra of the radial velocity at two locations in the main chamber are examined for the case with $C_{r}=0.325$. Looking at the spectra in Figure 15 one can see that there is a relatively broad range of frequencies present on the axis (for the inner vortex core) whereas the peaks are much weaker for the off-axis location. The peak with the maximum amplitude for the spectrum on the central axis is found at $87 \mathrm{~Hz}$, which in terms of Strouhal number equals to 0.29. For the off-axis spectrum the largest and second largest amplitudes are given at $162 \mathrm{~Hz}$ and $167 \mathrm{~Hz}$, i.e. Strouhal numbers of 0.54 and 0.56 , respectively. In general, the spectral content is seen to get richer around the center axis of the chamber as the contraction ratio gets smaller. In addition, the frequencies, as might be expected, are pushed towards higher values.

To further investigate the inner and outer vortex regions, Proper Orthogonal Decomposition (POD) [16, 17], and Dynamic Mode Decomposition (DMD) $[18,19]$ analyses are carried out. Two hundred snapshots of the 
whole three dimensional velocity field are used for each modal decomposition (DMD and POD). For the POD analysis the mean of the velocity fields are subtracted. Primary focus is placed on the two extreme cases (the smallest exhaust pipe, $C_{r}=0.325$, and no exhaust pipe, $C_{r}=1$ ). Iso-surfaces of the radial components of two DMD modes and four POD modes for the case with the smallest exhaust pipe are given in Figure 16 and Figure 17, respectively. The bright and dark regions correspond to values of opposite sign. Note that the spectral transforms discussed above use (point) data from a larger time interval, as well as a much smaller time step, compared with the mode analyses.

In order to understand the spatial shape of certain frequency modes, a DMD analysis is carried out for two frequencies. These two modes correspond to the spiraling modes with azimuthal wavenumbers $|m|=1$ and $|m|=2$. The $|m|=1$ mode has a low frequency, in part due to its low $|m|$ value, and represents a displacement of the vortex core. Since the mode is spinning, the displacement keeps oscillating along different directions. Because of the small diameter of the inner vortex core compared with the width of the chamber, which provides the vortex core with a large region to move around, the dipole-type flow mode, as seen from the DMD and POD modes, show a strong presence in the main chamber. As the geometric constriction gets more substantial similar modes of this type begin to appear, covering a larger range of frequencies, and an increased activity is seen in the pilot chamber. Flow structures with quadrupole character on the other hand start to play a larger role in the exhaust pipe, as well as away from the central axis in the main chamber. The quadrupole structure represents the simplest deformation, or squeezing, of the vortex core/ring. This explains its prevalence under more spatially constrained situations, such as for the vortex core inside the exhaust pipe, shown by the POD modes. On the other hand, the high frequency quadrupole mode inside the chamber, given by the DMD analysis, is a result of the outer vortex ring being constrained by the lateral walls of the chamber. This helical quadrupole mode is co-rotating and counter-winding (with respect to the mean flow). In contrast, the quadrupole structures in the exhaust pipe, as shown by the POD modes, are winding in the same general direction as the mean flow. As seen from the POD modes, the most energy containing structures for $C_{r}=0.325$ involve displacements and deformations of the inner vortex core.

Radial components of two DMD modes and four POD modes for the case without an exhaust pipe $\left(C_{r}=1\right)$ are given in Figure 18 and Figure 

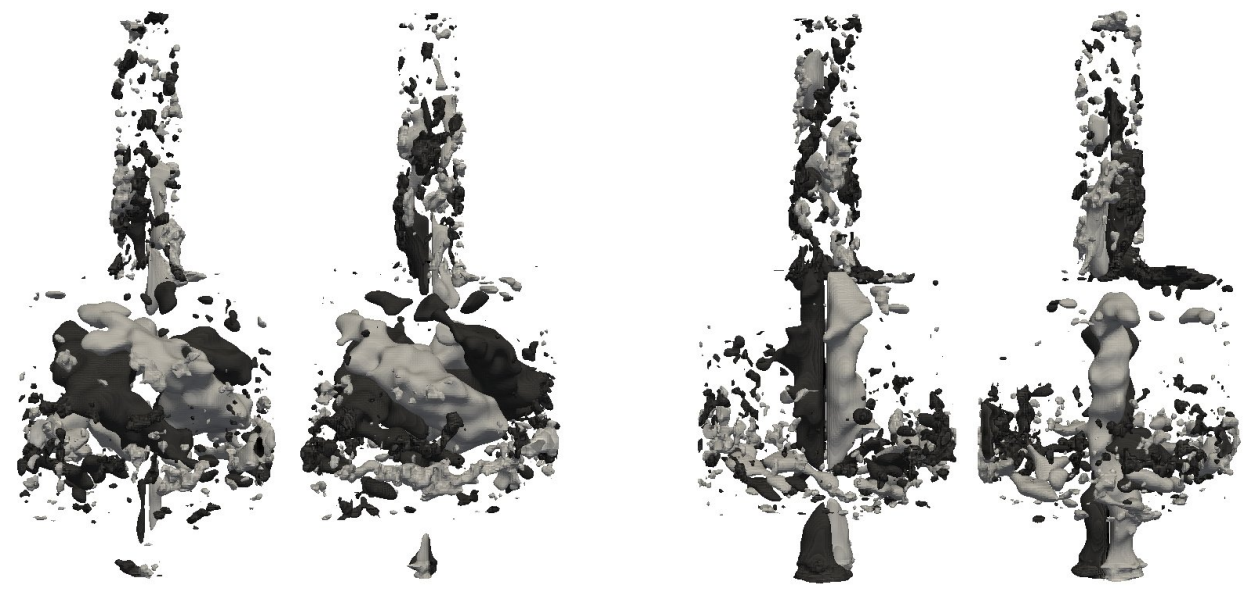

Figure 16: DMD modes for the $C_{r}=0.325$ case. Isosurfaces of the radial components of the real and imaginary parts of the modes are shown. Left: mode oscillating at $167 \mathrm{~Hz}$ corresponding to $\mathrm{St}=0.56$, right: mode oscillating at $87 \mathrm{~Hz}$ corresponding to $\mathrm{St}=0.29$.
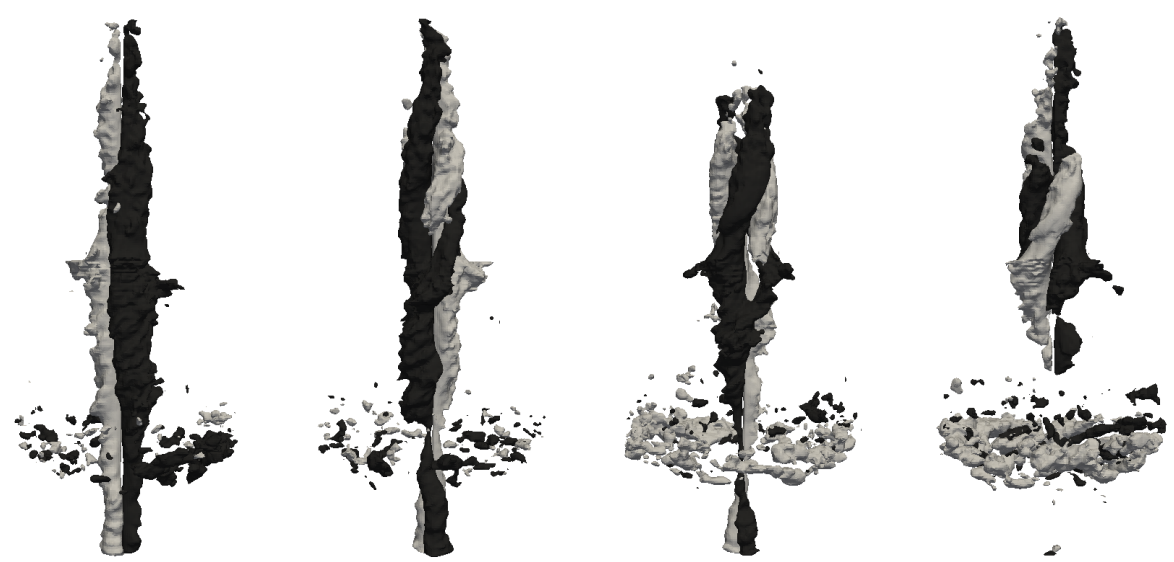

Figure 17: POD modes for the $C_{r}=0.325$ case. Iso-surfaces of the radial components of the modes are shown. The first four modes are given, counted from left to right. 

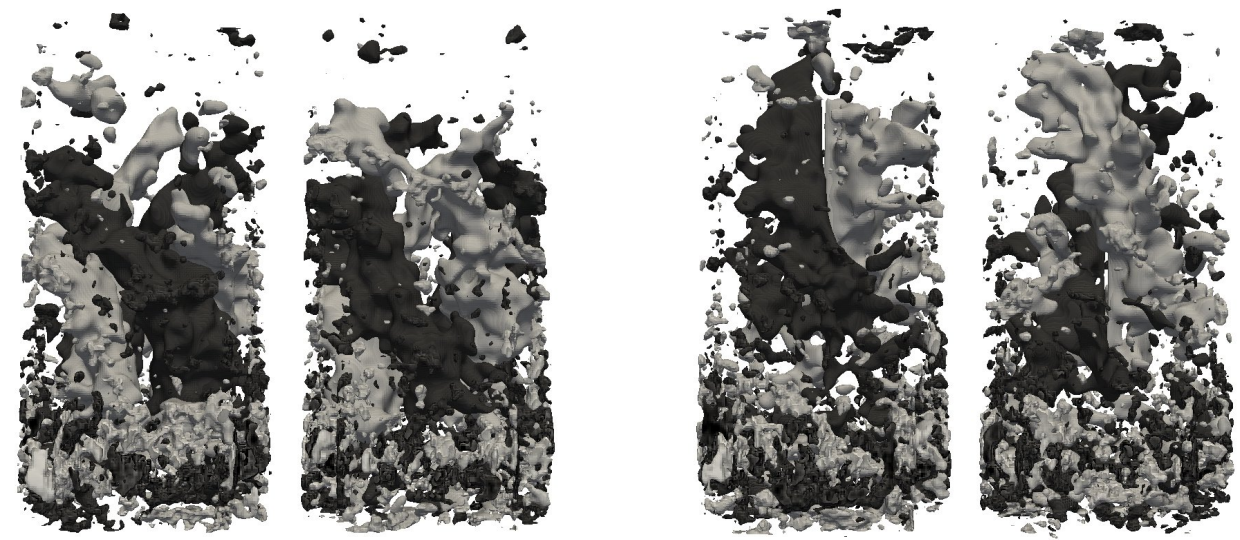

Figure 18: DMD modes for the $C_{r}=1$ case. Isosurfaces of the radial components of the real and imaginary parts of the modes are shown. Left: mode oscillating at $96 \mathrm{~Hz}$ corresponding to $\mathrm{St}=0.32$, right: mode oscillating at $41 \mathrm{~Hz}$ corresponding to $\mathrm{St}=0.13$.

19, respectively. As seen from the POD analysis, the most energetic modes correspond to quadrupole deformations of the outer vortex ring, which is clearly in contrast with the results from $C_{r}=0.325$. The first and second POD modes are of a helical quadrupole type, whereas the third and fourth have a helical dipole character. The pairs are essentially rotated versions of one another, reflecting the rotational motion of the modes. Note that the POD analysis is able to separate the two flow modes (dipole and quadrupole). The strong peak for the spectra in the upper part of Figure 20, using data from a point far downstream on the central axis $(Z / L=1.75)$, is found at $40 \mathrm{~Hz}$, which corresponds to a Strouhal number of 0.13 . The corresponding dipole mode appears to play a dominant role along the center of the chamber. It should be noted that the mode with the dipole character is much more spread out compared with the case with the smallest exhaust pipe diameter, which results from the larger inner vortex core, and that there is a suppressed activity in the pilot chamber. The spectrum in Figure 20 is extracted from data taken in the middle of the main chamber $(Z / L=0.75)$ on an off-axis location $(r / R=1.84)$. Similar to the $C_{r}=0.325$ case the peaks of the power spectra densities are much weaker in the off-axis locations than those on the center axis, which is owing to the lack of dynamic PVC motion in the off-axis regions. 

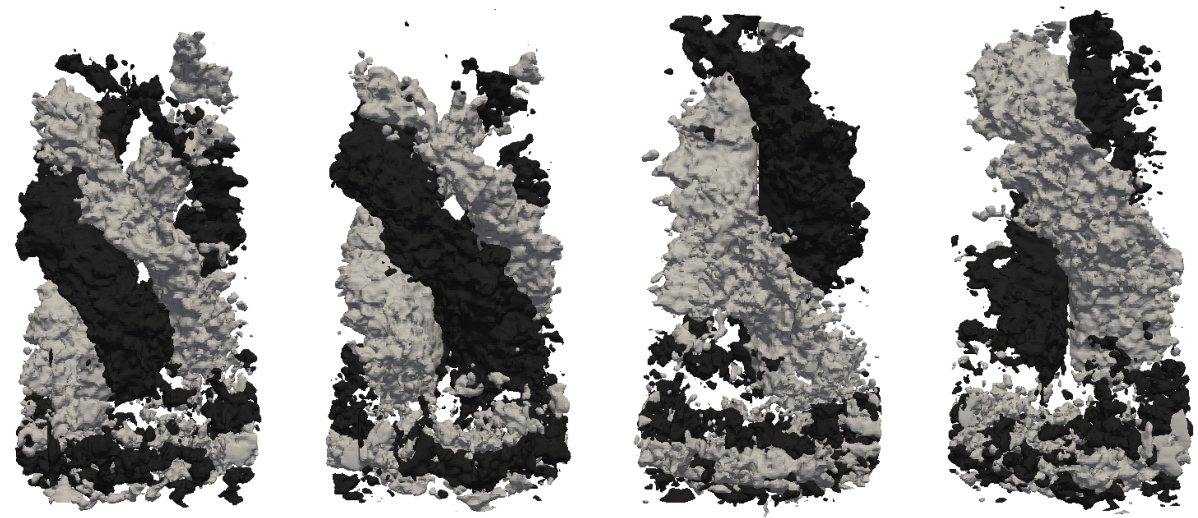

Figure 19: POD modes for the $C_{r}=1$ case. Isosurfaces of the radial components of the modes are shown. The first four modes are given, counted from left to right.
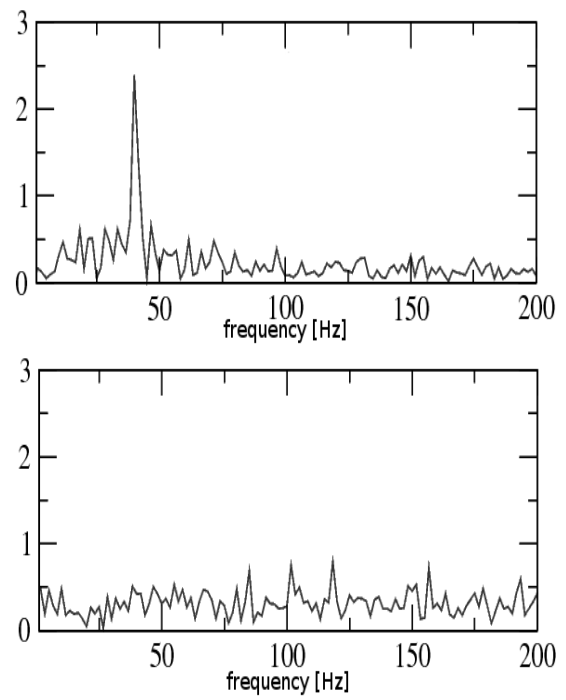

Figure 20: Power spectral densities for the case without an exhaust pipe. The spectra are derived from a radial velocity component. The top spectra stem from a location on the axis of the chamber at a downstream location, $Z / L=1.75$; the bottom spectra are from an off-center location $(X / R=1.84)$, situated in the middle of the chamber $(Z / L=0.75)$. 


\section{Conclusions}

The effects of outlet contraction on the structures of isothermal incompressible turbulent swirling flows in a model combustion chamber are studied using large eddy simulation. It is found that outlet contraction can exert a significant impact on the vortex breakdown structure and precessing vortex core. For the flow configuration under consideration there is inherently a corner recirculation zone formed owing to the sudden expansion of the main chamber geometry. Furthermore, due to the annular flow and the existence of a bowl for hosting the pilot flame there is a central recirculation zone. Under the studied high swirl number condition (swirl number of 1.4), if there is no outlet contraction, the central recirculation zone is large, and extends through the entire main chamber to the outlet. Around the axis of the main chamber there is a vortex core, in which the fluid is undergoing helical motion towards the upstream regions of the main chamber. The effect of adding an outlet contraction can be summarized as follows:

- By incorporating a contraction at the chamber outlet, the corner recirculation zone is seen not to be sensitive to the outlet geometry; however, the central recirculation zone is shown to be greatly affected by the outlet contraction. The center recirculation zone is split into two, one in the main chamber and one in the exhaust pipe; when the outlet area is decreased further the recirculation zone in the exhaust pipe is expelled outside the chamber.

- The outlet contraction enhances the strength of the inner vortex core. With smaller outlet area the diameter of the vortex core is slightly decreased and the tangential velocity significantly increased. Within the vortex core the radial velocity is rather small and the tangential velocity resembles that of solid body rotation. When the contraction outlet area becomes smaller, the inner vortex core becomes more energetic and the shape of the core becomes bent and twisted. The core precesses at a low frequency.

- There is a critical contraction ratio such that for the contraction ratio larger than the critical value the center recirculation zone in the main chamber is of central bubble type with flow reversal on the axis of the main chamber. When the contraction ratio is smaller than the critical value, the central recirculation zone takes a toroidal shape with 
the flow around the axis directed towards the chamber outlet. The transition from one type of recirculation to the other when varying the contraction ratio is not an abrupt process. No hysteresis effect is observed when successively varying the initial state of the simulations at different contraction ratios.

- Time dependence, in the form of large scale coherent structures, is captured using mode decompositions, DMD and POD, of the velocity fields. Two types of flow modes are distinguished, those of dipole character and those of quadrupole character. A physical background for these modes is presented and it is related to the level of confinement of the inner vortex core and outer vortex ring. The relative strength of the displacement and/or deformation of these different regions, basically involving solid body rotation, depend on the contraction ratio. For all the cases the flow field show double layer. DMD analysis of the two cases (with $C_{r}=0.325$ and $C_{r}=1$, respectively) shows that the inner layer are related to a low frequency, whereas the outer layer is related to a higher frequency.

- The fundamental effect of an outlet contraction can be explained through the budget terms in the momentum equations. The dominant terms in the momentum equation along the radial direction are the pressure gradient and the centrifugal force. Due to the enhancement of the tangential velocity in the center vortex core the centrifugal force is enhanced, which in turn enhances the gradient of pressure in the radial direction as well as in the axial direction. The low pressure center is on the axis of the main chamber and it moves towards the contraction plane when the contraction area decreases which essentially sucks in the fluid from the upstream. This stops the recirculation of fluid on the chamber axis from the chamber downstream to upstream, and eventually the fluid on the chamber axis flows towards the outlet.

\section{Acknowledgments}

This work was financially supported by the Swedish Research Council (VR) and partly by China Scholarship Council (CSC). The work was carried out at the Division of Fluid Mechanics, Department of Energy sciences, Faculty of Engineering LTH, Lund University, Sweden. The simulations have 
been conducted on Swedish National Infrastructure for Computing (SNIC) in Lund University (LUNARC) and Royal Institute of Technology (PDC).

[1] P. Weigand, W. Meier, X. Duan, W. Stricker, M. Aigner, Investigations of swirl flames in a gas turbine model combustor: I. flow field, structures, temperature, and species distributions, Combustion and Flame 144 (2006) 205-224.

[2] M. P. Escudier, J. Bornstein, N. Zehnder, Observations and LDA measurements of confined turbulent vortex flow, Journal of Fluid Mechanics 98 (1980) 49-63. doi:10.1017/S0022112080000031.

[3] R. Orbay, K. Nogenmyr, J. Klingmann, X. Bai, Swirling turbulent flows in a combustion chamber with and without heat release, Fuel 104 (0) (2013) 133 - 146. doi:10.1016/j.fuel.2012.09.023.

[4] P. Wang, X. S. Bai, M. Wessman, J. Klingmann, Large eddy simulation and experimental studies of a confined turbulent swirling flow, Physics of Fluids 16 (9) (2004) 3306-3324. doi:10.1063/1.1769420.

[5] P. Wang, X. Bai, Large eddy simulations of turbulent swirling flows in a dump combustor: A sensitivity study, International Journal for Numerical Methods in Fluids 47 (2) (2005) 99-120.

[6] T. Benjamin, Theory of the vortex breakdown phenomenon, Journal of Fluid Mechanics 14 (1962) 593-629.

[7] H. Squire, Analysis of the 'vortex breakdown' phenomenon, part i, Report No. 102, Aeronautics Department, Imperial College, London.

[8] M. Escudier, J. Keller, Recirculation in swirling flow: A manifestation of vortex breakdown., AIAA journal 23 (1) (1985) 111-116.

[9] M. Escudier, A. Nickson, R. Poole, Influence of outlet geometry on strongly swirling turbulent flow through a circular tube, Physics of Fluids 18 (12) (2006) 125103.

[10] C. Speziale, others, B. Younis, S. Berger, Analysis and modelling of turbulent flow in an axially rotating pipe, Journal of Fluid Mechanics 407 (2000) 1-26. doi:10.1017/S0022112099007600. 
[11] S. Pope, Ten questions concerning the large-eddy simulation of turbulent flows, New Journal of Physics 6 (2004) $1-24$.

[12] L. Selle, G. Lartigue, T. Poinsot, R. Koch, K.-U. Schildmacher, W. Krebs, B. Prade, P. Kaufmann, D. Veynante, Compressible large eddy simulation of turbulent combustion in complex geometry on unstructured meshes, Combustion and Flame 137 (4) (2004) 489 - 505. doi:10.1016/j.combustflame.2004.03.008.

[13] B. Wegner, A. Maltsev, C. Schneider, A. Sadiki, A. Dreizler, J. Janicka, Assessment of unsteady rans in predicting swirl flow instability based on les and experiments, International Journal of Heat and Fluid Flow 25 (3) (2004) 528-536.

[14] D. A. Nickolaus, C. E. Smith, Analysis of highly swirled, turbulent flows in dump combustor with exit contraction, ASME Turbo Expo 2005: Power for Land, Sea, and Air 2 (2005) 97-110.

[15] A. Escue, J. Cui, Comparison of turbulence models in simulating swirling pipe flows, Applied Mathematical Modelling 34 (10) (2010) 2840 - 2849. doi:10.1016/j.apm.2009.12.018.

[16] J. Lumley, Stochastic tools in turbulence, Academic Press.

[17] L. Sirovich, Turbulence and the dynamics of coherent structures, part i: Coherent structures, Q. Appl. Math. 45 (1987) 561 - 590.

[18] C. Rowley, I. Mezic, S. Bagheri, P. Schlatter, D. Henningson, Spectral analysis of nonlinear flow, J. Fluid Mech. 641 (2009) 115 - 127.

[19] P. Schmid, Dynamic mode decomposition of numerical and experimental data, J. Fluid Mech. 656 (2010) 5 - 28.

[20] J. Bourgouin, J. Moeck, S. Durox, S. Candel, Sensitivity of swirling flows to small changes in the swirler geometry, Comp. Rend. Mec. 341 (2013) $211-219$.

[21] H. Carlsson, C. Carlsson, L. Fuchs, X. Bai, Large eddy simulation and extended dynamic mode decomposition of flow-flame interaction in a lean premixed low swirl stabilized flame, Flow Turbulence and Combustion 93 (2014) $505-519$. 
[22] J. Smagorinsky, General circulation experiments with the primitive equations, i. the basic experiment, Monthly Weather Review 91 (3) (1963) 99-164.

[23] J. Deardorff, Stratocumulus-capped mixed layers derived from a threedimensional model, Boundary-Layer Meteorology 18 (4) (1980) 495-527. doi:10.1007/BF00119502.

[24] U. Schumann, Subgrid scale model for finite difference simulations of turbulent flows in plane channels and annuli, Journal of Computational Physics 18 (1975) 376-404.

[25] S. Menon, N. Patel, 'subgrid modeling for simulation of spray combustion in large-scale combustors, AIAA Journal 44 (2006) 709-723.

[26] M. Germano, U. Piomelli, P. Moin, W. H. Cabot, A dynamic subgridscale eddy viscosity model, Physics of Fluids A: Fluid Dynamics 3 (7) (1991) 1760-1765. doi:10.1063/1.857955.

[27] D. K. Lilly, A proposed modification of the germano subgrid-scale closure method, Physics of Fluids A: Fluid Dynamics 4 (3) (1992) 633-635.

[28] L. Davidson, Large eddy simulation: a dynamic one-equation subgrid model for three-dimensional recirculating flow, 11th Int. Symp. on Turb. Shear Flow 3 (9) (1997) 26.1-26.6.

[29] Openfoam user's guide, CFD Direct Ltd, Caversham, United Kingdom.

[30] J. Beér, N. Chigier, Combustion aerodynamics, Krieger Publishing Company, Malabar, FL, USA.

[31] U. Piomelli, E. Balaras, Wall-layer models for large-eddy simulations, Annu. Rev. Fluid Mech. 34 (2002) 349-374.

[32] P. Schmitt, T. Poinsot, B. Schuermans, K. Geigle, Lare-eddy simulation and experimental study of heat transfer, nitric oxide emissions and combustion instability in a swirler turbulent high-pressure burner, Journal of Fluid Mechanics 570 (2007) 17-46.

[33] R. Yu, X. Bai, A semi-implicit scheme for large eddy simulation of piston engine flow and combustion, Int. J. Numer. Meth. Fluids 71 (2013) 1340 . 
[34] T. Sarpkaya, On stationary and travelling vortex breakdowns, Journal of Fluid Mechanics 45 (1971) 545-559. doi:10.1017/S0022112071000181.

[35] P. Billant, J.-M. Chomaz, P. Huerre, Experimental study of vortex breakdown in swirling jets, Journal of Fluid Mechanics 376 (1998) 183219. doi:10.1017/S0022112098002870.

[36] P. Escudier, J. Keller, Vortex breakdown: A two-stage transition, Defense Technical Information Center, Brown Boveri Research Center Baden (Switzerland),

[37] M. Escudier, Vortex breakdown: Observations and explanations, Progress in Aerospace Sciences 25 (2) (1988) 189 - 229. doi:10.1016/0376-0421(88)90007-3.

[38] M. G. Hall, Vortex breakdown, Annu. Rev. Fluid Mech. 4 (1972) 195218.

[39] L. Facciolo, N. Tillmark, A. Talamelli, H. Alfredsson, A study of swirling turbulent pipe and jet flows, Physics of Fluids 19 (2007) 035105. 